\title{
The asymptotic cone of Teichmüller space and thickness
}

\author{
HAROLD SULTAN
}

\begin{abstract}
We study the asymptotic geometry of Teichmüller space equipped with the WeilPetersson metric. In particular, we provide a characterization of the canonical finest pieces in the tree-graded structure of the asymptotic cone of Teichmüller space along the same lines as a similar characterization for right angled Artin groups and for mapping class groups. As a corollary of the characterization, we complete the thickness classification of Teichmüller spaces for all surfaces of finite type, thereby answering questions of Behrstock, Druțu and Mosher, and Brock and Masur. In particular, we prove that Teichmüller space of the genus-two surface with one boundary component (or puncture) is the only Teichmüller space which is thick of order two.
\end{abstract}

30F60, 20F69; 20F65, 20F67

\section{Introduction}

In the setting of spaces of non-positive curvature, Euclidean and hyperbolic space represent the two extremes. In this paper we will see that the geometries of Teichmüller spaces for various surfaces provide natural examples of non-positively curved spaces that non-trivially interpolate between these two ends of the spectrum of non-positively curved spaces. In particular, the geometry of Teichmüller spaces includes on the one hand examples of hyperbolic and strongly relatively hyperbolic metric spaces, and on the other hand thick of order one and thick of order two metric spaces. The example of a Teichmüller space which is thick of order two is a novelty of this paper. Moreover, the tools developed in this paper for proving the aforementioned results are based on novel techniques used to study the asymptotic cones of spaces, and may have more general applications.

For $S$ a surface of finite type, the Teichmüller space $\mathcal{T}(S)$ is a classical space which parameterizes isotopy classes of hyperbolic structures on $S$. In the literature there are various natural metrics with which Teichmüller space can be equipped. Hereinafter, we always consider $\mathcal{T}(S)$ with the Weil-Petersson metric. The Weil-Petersson metric on $\mathcal{T}(S)$ is a complex-analytically defined Riemannian metric of variable non-positive 
curvature. While the space is not complete, its completion is a CAT(0) metric space; see Wolpert [23]. The large scale geometry of Teichmüller space has been an object of recent interest, especially within the circles of ideas surrounding Thurston's ending lamination conjecture. In this context, the pants complex $\mathcal{P}(S)$, a combinatorial complex associated to a hyperbolic surface $S$, becomes relevant. Specifically, by a groundbreaking theorem of Brock [7], $\mathcal{P}(S)$ is quasi-isometric to $\mathcal{T}(S)$. Accordingly, in order to study large scale geometric properties of Teichmüller space, it suffices to study the pants complex of a surface. Recent results such as Behrstock [1], Behrstock and Minsky [6], Brock and Farb [8], Brock and Masur [9] and Brock, Masur and Minsky [10; 11], among others, can be viewed from this perspective. Similarly, all of the results of this paper regarding the coarse structure of the pants complex should be interpreted as coarse results regarding Teichmüller space.

In recent years, study of asymptotic cones has proven extremely fruitful in considering the coarse geometry of groups and spaces. See for instance Behrstock, Druțu and Sapir [4], Druțu [12] and Druțu and Sapir [14]. One aspect in common to the aforementioned studies of asymptotic cones is interest in cut-points, namely single points whose removal disconnects the asymptotic cone. The general theme is that cut-points in asymptotic cones correspond to a weak form of hyperbolicity in the underlying space. One of the highlights of the paper is a characterization of when two points in the asymptotic cone of Teichmüller space are separated by a cut-point, see Theorem 4.8.

On the one hand, it is shown in Behrstock [1] that in the asymptotic cone of Teichmüller space, every point is a global cut-point. On the other hand, for high enough complexity surfaces, Teichmüller space has natural quasi-isometrically embedded flats, or quasiflats; see Behrstock and Minsky [6], Brock and Farb [8] and Masur and Minsky [19]. In turn, this ensures the existence of non-trivial subsets of the asymptotic cone without internal cut-points. Putting things together, the asymptotic cone of Teichmüller space is a tree-graded space. In such a setting, there are canonically defined finest pieces of the tree-graded structure, which are defined to be maximal subsets of the asymptotic cone subject to the condition that no two points in a finest piece can be separated by the removal of a point. A highlight of this paper is the following theorem that characterizes the finest pieces in tree-graded structure of the asymptotic cone of Teichmüller space.

Theorem 4.8 Let $S=S_{g, n}$, and let $\mathcal{P}_{\omega}(S)$ be any asymptotic cone of $\mathcal{P}(S)$. Then for all $a_{\omega}, b_{\omega} \in \mathcal{P} \omega(S)$, the following are equivalent:

(1) No point separates $a_{\omega}$ and $b_{\omega}$, or equivalently $a_{\omega}$ and $b_{\omega}$ are in the same canonical finest piece.

(2) In any neighborhood of $a_{\omega}, b_{\omega}$, respectively, there are $a_{\omega}^{\prime}, b_{\omega}^{\prime}$, with representative sequences $\left(a_{n}^{\prime}\right),\left(b_{n}^{\prime}\right)$, such that $\lim _{\omega} d_{\mathbb{S}(S)}\left(a_{n}^{\prime}, b_{n}^{\prime}\right)<\infty$. 
The characterization of finest pieces in Theorem 4.8 is given in terms of the complex of separating multicurves $\mathbb{S}(S)$ which encodes information about the natural product structures in the pants complex. The complex of separating multicurves will be defined and explored in Section 3. The proof of Theorem 4.8 relies heavily on a notion of structurally integral corners to be developed in Section 4.1. Roughly speaking, a structurally integral corner is a point in the asymptotic cone whose removal disconnects particular natural product regions. Structurally integral corners only exist for lowcomplexity surfaces. Theorem 4.8 should be compared with [2, Theorem 4.6] and [5, Theorem 7.9], where similar characterizations of the finest pieces are proven for right angled Artin groups and mapping class groups, respectively.

Later, we focus in particular on the Teichmüller space of the surface $S_{2,1}$. For "small" complexity surfaces which don't admit any non-trivial separating curves, Brock and Farb [8] prove that $\mathcal{T}(S)$ is hyperbolic. Similarly, for "medium" complexity surfaces, which admit non-trivial separating curves, yet have the property that any two separating curves intersect, Brock and Masur [9] prove that $\mathcal{T}(S)$ is relatively hyperbolic. Finally, for all the remaining "large" complexity surfaces excluding $S_{2,1}$, whose complexes of separating multicurves only have a single infinite-diameter connected component, the combined work of Behrstock [1] Brock and Masur [9] implies that the Teichmüller spaces of these surfaces are not relatively hyperbolic and in fact are thick of order one. However, unlike all other surfaces of finite type, the surface $S_{2,1}$ has the peculiar property that it is "large enough" such that it admits disjoint separating curves, although "too small" such that the complex of separating multicurves has infinitely many infinite diameter connected components. As we will see, this phenomenon makes the study of $\mathcal{T}\left(S_{2,1}\right)$ quite rich.

Theorem $5.7 \mathcal{T}\left(S_{2,1}\right)$ is thick of order two.

Notably, Theorem 5.7 completes the thickness classification of the Teichmüller spaces of all surfaces of finite type. Moreover, among all surfaces of finite type, $S_{2,1}$ is the only surface that is thick of order two.

Acknowledgements This paper comprises a portion of the author's doctoral thesis [21]. My sincerest gratitude goes to my advisors Jason Behrstock and Walter Neumann for all of their invaluable guidance and extremely helpful advice throughout my research and specifically with regard to this paper. They selflessly encouraged, advised, directed, and challenged me in my research. I would also like to thank Lee Mosher, Saul Schleimer, and the referee for many useful suggestions and comments. 


\section{Background}

\subsection{Curves and essential subsurfaces}

Let $S=S_{g, n}$, by any orientable surface of finite type. That is, $S$ is a genus- $g$ surface with $n$ boundary components (or punctures). The complexity of $S$, denoted $\xi(S)$, is defined to be $3 g-3+n$. In this paper while we will always refer to surfaces with boundary components, the same results hold mutatis mutandis for surfaces with punctures.

An isotopy class of simple closed curve $\gamma$ on a surface $S$ is called essential as long as it doesn't bound a disk, a once punctured disk, or an annulus. Since we will always consider curves up to isotopy, we can always assume that their intersections are transverse and cannot be removed. A multicurve is a set of disjoint non-parallel curves. An essential subsurface $Y$ of a surface $S$ is a subsurface $Y \subseteq S$ such that $Y$ is a union of (not necessarily all) complementary components of a multicurve. We always consider essential subsurfaces up to isotopy classes. Furthermore, we always assume every connected component of every essential subsurface $Y \subset S$ has complexity at least one. In particular, annuli or pairs of pants are not considered essential subsurfaces and do not appear as connected components of essential subsurfaces. For a fixed surface $S$, let $\mathcal{E}(S)$ denote the set of all connected essential subsurfaces of $S$.

Given any essential subsurface $Y$ we define the essential complement of $Y$, denoted $Y^{c}$, to be the maximal (in terms of containment) essential subsurface in the complement $S \backslash Y$ if such an essential subsurface exists, and to be the empty set otherwise. An essential subsurface $Y$ is called a separating essential subsurface if the complement $S \backslash Y$ contains an essential subsurface, or equivalently $Y^{c}$ is non-trivial. The reason for the name separating essential subsurface is due to that the fact that $Y$ is a separating essential subsurface if and only if the boundary $\partial Y$ is a separating multicurve, an object we will consider at length in Section 3. All other essential subsurfaces which are not separating essential subsurfaces, are defined to be non-separating essential subsurfaces. For example, if $Y$ is an essential subsurface such that the complement $S \backslash Y$ consists of a disjoint union of annuli and pairs of pants, then $Y$ is a non-separating essential subsurface. Let the subsets $\mathcal{S E}(S), \mathcal{N E}(S) \subset \mathcal{E}(S)$ denote the sets of all connected separating, non-separating essential subsurfaces of $S$, respectively.

An essential subsurface $Y$ is proper if it is not all of $S$. If two essential subsurfaces $W, V$ have representatives which can be drawn disjointly on a surface they are said to be disjoint. On the other hand, we say $W$ is nested in $V$, denoted $W \subset V$, if $W$ has a representative which can be realized as an essential subsurface inside a representative of the essential subsurface $V$. If $W$ and $V$ are not disjoint, yet neither essential subsurface is nested in the other, we say that $W$ overlaps $V$, denoted $W \pitchfork V$. In 
general, if two essential subsurfaces $W, V$ either are nested or overlap, we say that the surfaces intersect each other. In such a setting we define the essential intersection, denoted $W \cap V$, to be the maximal essential subsurface which is nested in both $W$ and $V$, if such an essential subsurface exists, and the emptyset otherwise. Note that $W \cap V$ may be trivial even if the essential subsurfaces $W, V$ are not disjoint, as the intersection $W \cap V$ may be supported in a subsurface which is not essential. Similarly, the essential complement of $V$ in $W$, denoted $W \backslash V$, is defined to be the maximal essential subsurface in $(S \cap W) \backslash Y$ if such an essential subsurface exists, and to be the empty set otherwise.

\subsection{Curve and pants complex}

For any surface $S$ with positive complexity, the curve complex of $S$, denoted $\mathcal{C}(S)$, is the simplicial complex obtained by associating a $0-$ cell to each curve, and more generally a $k$-cell to each multicurve with $k+1$ elements. In the special case of low complexity surfaces which do not admit disjoint curves, we relax the notion of adjacency to allow edges between vertices corresponding to curves which intersect minimally on the surface. $\mathcal{C}(S)$ is a locally infinite, infinite-diameter, $\delta$-hyperbolic metric space [18], pants decompositions are maximal multicurves. Equivalently, a pants decomposition is a multicurve whose complement consists of a disjoint union of pairs of pants or copies of $S_{0,3}$.

The pants complex $\mathcal{P}(S)$ has vertices corresponding to different pants decompositions of the surface up to isotopy, and edges between two vertices when the two corresponding pants decompositions differ by a so-called elementary pants move. Specifically, two pants decompositions of a surface differ by an elementary pants move if the two decompositions differ in exactly one curve and inside the unique connected complexity one essential subsurface in the complement of all the other agreeing curves of the pants decompositions (topologically either an $S_{1,1}$ or an $S_{0,4}$ ) the differing curves intersect minimally (namely, once if the connected complexity one essential subsurface is $S_{1,1}$ and twice if the connected complexity one essential subsurface is $\left.S_{0,4}\right) . \mathcal{P}(S)$ is connected [15], and moreover after endowing it with the graph metric, $\mathcal{P}(S)$ is quasi-isometric to Techimüller space equipped with the Weil-Petersson metric [7]. Accordingly, in order to prove quasi-isometric invariant properties for Techimüller space equipped with the Weil-Petersson metric it suffices to prove them for $\mathcal{P}(S)$.

\subsection{Asymptotic cones}

Given a sequence of points $\left(x_{i}\right)$ and an ultrafilter $\omega$, the ultralimit of $\left(x_{i}\right)$, denoted $\lim _{\omega} x_{i}$, is defined to be $x$ if for any neighborhood $U$ of $x$, the set $\left\{i \mid x_{i} \in U\right\} \in \omega$. That is, $\omega$ almost surely (or $\omega-a s) x_{i} \in U$. 
Given any set $S$ and an ultrafilter $\omega$, we define the ultrapower of $S$, denoted $S^{\omega}$, as sequences $\bar{s}$ or $\left(s_{i}\right)$ under the equivalence relation $\bar{s} \sim \overline{s^{\prime}} \Longleftrightarrow \omega$-as $s_{i}=s_{i}^{\prime}$. Elements of the ultrapower will be denoted $s^{\omega}$ and their representative sequences will be denoted by $\bar{s}$ or $\left(s_{i}\right)$. By abuse of notation we will sometimes denote elements of the ultrapower and similarly elements of the asymptotic cone by their representative sequences.

For a metric space $(X, d)$, we define the asymptotic cone of $X$, relative to a fixed choice of ultrafilter $\omega$, a sequence of base points in the space $\left(x_{i}\right)$, and an unbounded sequence of positive scaling constants $\left(s_{i}\right)$, as follows:

$$
\text { Cone }_{\omega}\left(X,\left(x_{i}\right),\left(s_{i}\right)\right) \equiv \lim _{\omega}\left(X, x_{i}, d_{i}=\frac{d}{s_{i}}\right) .
$$

When the choice of scaling constants and base points are not relevant we denote the asymptotic cone of a metric space $X$ by $X_{\omega}$. Elements of asymptotic cones will be denoted $x_{\omega}$ with representatives denoted by $\bar{x}$ or $\left(x_{i}\right)$. For $\mathcal{P}(S)$ we denote Cone $_{\omega}\left(\mathcal{P}(S),\left(P_{i}^{0}\right),\left(s_{i}\right)\right)=\mathcal{P}_{\omega}(S)$. For a reference on asymptotic cones, see [13].

More generally, given a subset $Y \subset X$, and a choice of asymptotic cone $X_{\omega}$, throughout we will often consider the ultralimit of $\mathrm{Y}$, denoted $Y_{\omega}$, defined as

$$
Y_{\omega}=:\left\{y_{\omega} \in X_{\omega} \mid y_{\omega} \text { has a representative sequence }\left(y_{i}^{\prime}\right) \text { with } y_{i}^{\prime} \in Y \omega \text {-as }\right\} \text {. }
$$

In particular, when dealing with ultralimits we will always be considering the ultralimits as subsets contained inside an understood asymptotic cone. Furthermore, given a sequence of subspaces $Y_{i} \subset X$, we can similarly define the ultralimit $Y_{\omega}$. Based on the context it will be clear which type of ultralimit is being considered.

The next elementary lemma follows from the definition of $\omega$.

Lemma 2.1 If $A$ is a finite set, then any $\bar{\alpha} \in A^{\omega}$ is $\omega$-as constant. That is, there is a unique $a_{0}$ in $A$ such that $\left\{i \mid \alpha_{i}=a_{0}\right\} \in \omega$. In particular, $\left|A^{\omega}\right|=|A|$.

Recall that subsets $A, B \subset X$, are said to coarsely intersect, denoted $A \widehat{\cap} B \neq \varnothing$, if there is a positive constant $r$ such that any two elements in the collection of subsets $\left\{N_{R}(A) \cap N_{R}(B) \mid R \geq r\right\}$ have finite Hausdorff distance. Moreover, if $C \subset X$ has finite Hausdorff distance from any set $N_{R}(A) \cap N_{R}(B)$, then $C$ is the coarse intersection of the subsets $A$ and $B$. If $C$ has bounded diameter, we say the subsets $A$ and $B$ have bounded coarse intersection. Finally, we will prove a technical lemma relating asymptotic cones and coarse intersection.

Lemma 2.2 Let $A, B \subset X$ such that in all asymptotic cones $X_{\omega}$, the constant subsets $\left(A_{i}\right)=\bar{A}=A_{\omega}$ and $\left(B_{i}\right)=\bar{B}=B_{\omega}$ satisfy $\left|A_{\omega} \cap B_{\omega}\right| \leq 1$. Then $A$ and $B$ have bounded coarse intersection. 
Proof We will prove the contrapositive. There are two cases to consider, namely (1) $A, B$ have infinite diameter coarse intersection, and (2) $A, B$ have undefined coarse intersection. In case (1) it is trivial to construct an asymptotic cone $X_{\omega}$ with constant base point in the coarse intersection of $A, B$ such that $\left|A_{\omega} \cap B_{\omega}\right|$ is infinite.

In case (2), since $A, B$ have undefined coarse intersection, it follows that for all $r_{i}$, there are $R_{i}^{1}, R_{i}^{2}$ such that $Y_{i}^{1}=N_{R_{i}^{1}}(A) \cap N_{R_{i}^{1}}(B)$ and $Y_{i}^{2}=N_{R_{i}^{2}}(A) \cap N_{R_{i}^{2}}(B)$ have infinite Hausdorff distance. Without loss of generality, assume $R_{i}^{2}>R_{i}^{1}$. After replacing $Y_{i}^{1}$ and $Y_{i}^{2}$ with their closures, it follows that there is a point $y_{i}^{2} \in Y_{i}^{2}$ such that $d\left(y_{i}^{2}, Y_{i}^{1}\right)=s_{i}>2^{R_{i}^{2}}$. Let $y_{i}^{1}$ be the closest point in $Y_{i}^{1}$ to $Y_{i}^{2}$. Finally, consider the asymptotic cone $X_{\omega}=\operatorname{Cone}_{\omega}\left(X,\left(y_{i}^{1}\right),\left(s_{i}\right)\right)$. By construction the points $\left(y_{i}^{1}\right)=y_{\omega}^{1}$ and $\left(y_{i}^{2}\right)=y_{\omega}^{2}$ are distinct in $X_{\omega}$ (in fact they are precisely distance one apart) and moreover they are both contained in $A_{\omega} \cap B_{\omega}$, thereby completing the proof of the lemma.

\section{4 (Relative) hyperbolicity and thickness}

An important generalization of hyperbolicity is the notion of relative hyperbolicity. Informally, a metric space $X$ is relatively hyperbolic with respect to a collection of subsets $\mathcal{A}$, if when all of the subsets in $\mathcal{A}$ are collapsed to finite diameter sets, the resulting "electric space" $X / \mathcal{A}$ is hyperbolic. More specifically, we will use the following definition of strong relative hyperbolicity of a metric space due to [14] formulated in terms of asymptotic cones:

Definition 2.3 (Relatively hyperbolic) A metric space $(X, d)$ is said to be hyperbolic relative to a collection of peripheral subsets $\mathcal{A}$ if $X$ is asymptotically tree-graded, with respect to $\mathcal{A}$. That is:

(1) Every asymptotic cone $X_{\omega}$ is tree-graded with respect to the pieces $A_{\omega}$ for $A \in \mathcal{A}$. More specifically, the intersection of each pair of distinct pieces, $A_{\omega}, A_{\omega}^{\prime}$, has at most one point and every simple geodesic triangle (a simple loop composed of three geodesics) in $X_{\omega}$ lies in one piece $A_{\omega}$.

(2) $X$ is not contained in a finite radius neighborhood of any of the subsets in $\mathcal{A}$.

It should be noted that the second condition is not always required, and when it is required the above notion is then referred to as non-trivial or proper relative hyperbolicity.

In contrast to earlier concepts of hyperbolicity or relatively hyperbolicity, we have the following notion of thickness developed in [3]. 
Definition 2.4 (Thickness [9, Definition 17]) (1) A space $X$ is said to be thick of order zero if it is unconstricted, namely there exists some ultrafilter $\omega$ and some sequence of scalars $s_{i}$ such that for any sequence of basepoints the corresponding asymptotic cone $X_{\omega}$ has no cut-points. Moreover, $X$ satisfies the following nontriviality condition: there is a constant $c$ such that every $x \in X$ is distance at most $c$ from a bi-infinite quasi-geodesic in $X$.

(2) A space $X$ is said to be thick of order at most $n+1$ if there are subsets $P_{\alpha} \subset X$ that satisfy the following conditions:

(i) With their restricted metric from $\mathrm{X}$, the subsets $P_{\alpha}$ are uniformly thick of order at most $n$.

(ii) Almost everything There is a fixed constant $R_{1}$ such that $\bigcup_{\alpha} N_{R_{1}}\left(P_{\alpha}\right)=X$.

(iii) Chained together thickly For any subsets $P_{\alpha}, P_{\beta}$, there exists a sequence of subsets $P_{\alpha}=P_{\gamma_{1}}, \ldots, P_{\gamma_{n}}=P_{\beta}$ such that for some fixed constant $R_{2} \geq 0$, $\operatorname{diam}\left(N_{R_{2}}\left(P_{\gamma_{i}}\right) \bigcap N_{R_{2}}\left(P_{\gamma_{i+1}}\right)\right)=\infty$.

(3) A collection of metric spaces $\left\{X_{i}\right\}$ is called uniformly thick of order at most $n+1$ if a uniform $R_{1}, R_{2}$ can be taken in the above definition.

(4) A space $X$ is thick of order $n$ if $n$ is the lowest integer such that $X$ is thick of order at most $n$.

In this paper we will be focusing on $T(S)$, a CAT(0) space which is coarsely homogeneous (admits a coarsely transitive group action by isometries), and has extendable geodesics. Accordingly, by [22, Theorem 5.1], it follows that for our purposes, in the definition of a space $X$ being thick of order zero above we can replace $X$ being unconstricted with $X$ being wide, namely all asymptotic cones $X_{\omega}$ are without cutpoints.

In Section 5 we will often be interested in subspaces $Y \subset X$ which are thick of order zero. Namely, we say that a subspace $Y$ is thick of order zero if in every asymptotic cone $X_{\omega}$ the subset corresponding to the ultralimit $Y_{\omega}$ has the property that any two distinct points in $Y_{\omega}$ are not separated by a cut-point. Additionally, we require that $Y$ satisfies the non-triviality condition of every point being within distance $c$ from a bi-infinite quasi-geodesic in $Y$.

The following theorem of [3], which inspired the development of the notion of thickness, captures the contrasting relationship between hyperbolicity and thickness:

Theorem 2.5 [3, Corollary 7.9] A metric space $X$ which is thick of any finite order is not strongly relatively hyperbolic with respect to any subsets. 


\subsection{Subsurface projections}

Given a curve $\alpha \in \mathcal{C}(S)$ and a connected essential subsurface $Y \in \mathcal{E}(S)$ such that $\alpha$ intersects $Y$, we can define the projection of $\alpha$ to $2^{\mathcal{C}(Y)}$, denoted $\pi_{\mathcal{C}(Y)}(\alpha)$, to be the collection of vertices in $\mathcal{C}(Y)$ obtained in the following surgical manner. The intersection $\alpha \cap Y$ consists of either the curve $\alpha$, if $\alpha \subset Y$, or a non-empty disjoint union of arc subsegments of $\alpha$ with the endpoints of the arcs on boundary components of $Y$. In the former case we define the projection $\pi_{\mathcal{C}(Y)}(\alpha)=\alpha$. In the latter case, $\pi_{\mathcal{C}(Y)}(\alpha)$ consists of all curves obtained by the following process. If an $\operatorname{arc}$ in $\alpha \cap Y$ has both endpoints on the same boundary component of $\partial Y$, then $\pi_{\mathcal{C}(Y)}(\alpha)$ includes the curves obtained by taking the union of the arc and the boundary component containing the endpoints of the arc. Note that this yields at most two curves, at least one of which is essential. On the other hand, if an arc in $\alpha \cap Y$ has endpoints on different boundary components of $\partial Y$, then $\pi_{\mathcal{C}(Y)}(\alpha)$ includes the curve on the boundary of a regular neighborhood of the union of the arc and the different boundary components containing the end points of the arc. See Figure 1 for an example. Note that above we have only defined the projection $\pi_{\mathcal{C}(Y)}$ for curves intersecting $Y$, for all curves $\gamma$ disjoint from $Y$, the projection $\pi_{\mathcal{C}(Y)}(\gamma)=\varnothing$.

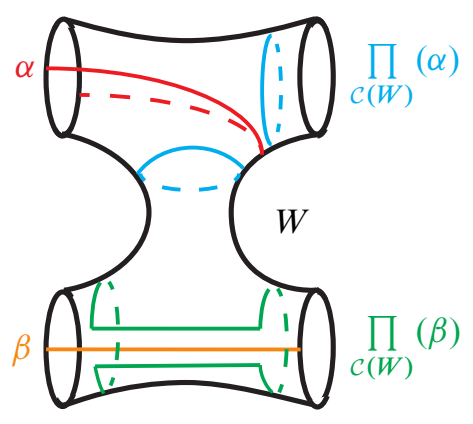

Figure 1: Performing surgery on arcs intersecting $W \subsetneq S$

In any context concerning the curve complex of an essential subsurface, $\mathcal{C}(Y)$ in order to avoid distractions we always assume that $Y \in \mathcal{E}(Y)$, ie the essential subsurface $Y$ is connected. To simplify notation, we write $d_{\mathcal{C}(Y)}\left(\alpha_{1}, \alpha_{2}\right)$ as shorthand for $d_{\mathcal{C}(Y)}\left(\pi_{\mathcal{C}(Y)}\left(\alpha_{1}\right), \pi_{\mathcal{C}(Y)}\left(\alpha_{2}\right)\right)$. In particular, this distance is only well-defined if $\alpha_{1}, \alpha_{2}$ intersect $Y$. Similarly, for $A \subset \mathcal{C}(S)$, we write $\operatorname{diam}_{\mathcal{C}(Y)}(A)$ as shorthand for $\operatorname{diam}_{\mathcal{C}(Y)}\left(\pi_{\mathcal{C}(Y)}(A)\right)$.

The following lemma ensures that the subsurface projection $\pi_{\mathcal{C}(Y)}$ defined above gives a coarsely well-defined projection $\pi_{\mathcal{C}(Y)}: \mathcal{C}(S) \rightarrow \mathcal{C}(Y) \cup \varnothing$. 
Lemma 2.6 [19, Lemma 2.2] For $\alpha$ any curve and any $Y \in \mathcal{E}(Y)$ the set of curves $\pi_{\mathcal{C}(Y)}(\alpha)$ has diameter bounded above by three. Hence, we have a coarsely well-defined subsurface projection map which by abuse of notation we refer to as $\pi_{\mathcal{C}(Y)}: \mathcal{C}(S) \rightarrow$ $\mathcal{C}(Y) \cup \varnothing$. In particular, if $\sigma$ is any connected path in $\mathcal{C}(S)$ of length $n$, and $Y$ is any connected subsurface such that every curve in the path $\sigma$ intersects $Y$, then $\operatorname{diam}_{\mathcal{C}(Y)}(\sigma) \leq 3 n$

The next theorem describes a situation in which subsurface projection maps geodesics in the curve complex to uniformly bounded diameter subsets in the curve complex of a connected essential subsurface.

Theorem 2.7 (Bounded geodesic image [19, Theorem 3.1]) Let $Y \in \mathcal{E}(S)$ be a connected proper essential subsurface of $S$, and let $g$ be a geodesic (segment, ray, or bi-infinite) in $\mathcal{C}(S)$ such that every curve corresponding to a vertex of $g$ intersects $Y$, then $\operatorname{diam}_{\mathcal{C}(Y)}(g)$ is uniformly bounded by a constant $K(S)$ depending only on the topological type of $S$.

In addition to projecting curves, we can similarly project multicurves. In particular, we can project pants decompositions of surfaces to essential subsurfaces. Specifically, for any essential subsurface $Y$ we have an induced coarsely well-defined projection map

$$
\pi_{\mathcal{P}(Y)}: \mathcal{P}(S) \rightarrow \mathcal{P}(Y) .
$$

The induced map is defined as follows. Beginning with any pair of pants $P \in \mathcal{P}(S)$ there is at least one curve $\alpha_{1} \in P$ intersecting $Y$. We then proceed to construct a pants decomposition of $Y$ inductively. As our first curve we simply pick any curve $\beta_{1} \in$ $\pi_{\mathcal{C}(Y)}\left(\alpha_{1}\right)$. Then, we consider the surface $Y \backslash \beta_{1}$ and notice that $\xi\left(Y \backslash \beta_{1}\right)=\xi(Y)-1$. Replace $Y$ by $Y \backslash \beta_{1}$ and repeat this process until the complexity is reduced to zero. At this point, the curves $\left\{\beta_{i}\right\}$ are a pants decomposition of the essential subsurface $Y$. Due to all the choice, the above process does not produce a unique pants decomposition. Nonetheless, as in Lemma 2.6 the map is coarsely well-defined and in fact is coarsely Lipschitz with uniform constants [1; 19].

The next lemma makes precise a sense in which distances under projections to curve complexes of overlapping surfaces are related to each other. Intuitively, the point is that the distance in one subsurface projection can be large only at the expense of the distance in all overlapping essential subsurfaces being controlled.

Lemma 2.8 (Behrstock inequality [1, Theorem 4.3; 17, Lemma 2.5]) For $S=S_{g, n}$, let $W, V \in \mathcal{E}(S)$ be such that $W \pitchfork V$. Then for all $P \in \mathcal{P}(S)$,

$$
\min \left(d_{\mathcal{C}(W)}(P, \partial V), d_{\mathcal{C}(V)}(P, \partial W)\right) \leq 10 .
$$


Utilizing the projection $\pi_{\mathcal{P}(Y)}: \mathcal{P}(S) \rightarrow \mathcal{P}(Y)$, for $\bar{Y} \in \mathcal{E}^{\omega}(S)$ we can define $\mathcal{P}_{\omega}(\bar{Y})$ to be the ultralimit of $\mathcal{P}\left(Y_{i}\right)$. It is clear that $\mathcal{P}_{\omega}(\bar{Y})$ is isomorphic to $\mathcal{P}_{\omega}(Y)$ for $Y$ an essential subsurface $\omega$-as isotopic to $Y_{i}$. Moreover, extending the coarsely welldefined Lipschitz projection $\pi_{\mathcal{P}(Y)}: \mathcal{P}(S) \rightarrow \mathcal{P}(Y)$ to the asymptotic cone, we have a Lipschitz projection

$$
\pi_{\mathcal{P}_{\omega}(\bar{Y})}: \mathcal{P}_{\omega}(S) \rightarrow \mathcal{P}_{\omega}(\bar{Y})
$$

\subsection{Tight geodesics and hierarchies}

A fundamental obstacle in studying geodesics in the curve complex stems from the fact that the 1-skeleton is locally infinite. In an effort to navigate this problem, Masur and Minsky [19] introduced a notion of tight multigeodesics, or simply tight geodesics, in $\mathcal{C}(S)$. Specifically, for $S$ a surface of finite type with $\xi(S) \geq 2$, a tight geodesic in $\mathcal{C}(S)$ is a sequence of simplices $\sigma=\left(w_{0}, \ldots, w_{n}\right)$ such that the selection of any curves $v_{i} \in w_{i}$ yields a geodesic in $\mathcal{C}(S)$ and moreover, for $1 \leq i \leq n-1$, the simplex $w_{i}$ is the boundary of the essential subsurface filled by the curves $w_{i-1} \cup w_{i+1}$. In the case of a surface $S$ with $\xi(S)=1$ every geodesic is considered tight. For $\sigma$ a tight geodesic as above, we use the notation $\left[w_{i}, w_{j}\right]=\left(w_{i}, \ldots, w_{j}\right)$ to refer to a subsegment of the tight geodesic. In [19] it is shown that any two curves in $\mathcal{C}(S)$ can be joined by a tight geodesic (and in fact there are only finitely many).

Using tight geodesics, in [19] a 2-transitive family of quasi-geodesics, with constants depending on the topological type of $S$, in $\mathcal{P}(S)$ called hierarchies, are developed. Since we are interested in paths in the pants complex as opposed to the marking complex, unless specified otherwise we use the term "hierarchies" to refer to what are in fact called "resolutions of hierarchies without annuli" in [19]. The construction of hierarchies which are defined inductively as a union of tight geodesics in the curve complexes of connected essential subsurfaces of $S$ is technical. For our purposes, it will suffice to record some of their properties in the following theorem. See [9, Definition 9] for a similar statement.

Theorem 2.9 (Hierarchies [19, Section 4]) For $S$ any surface of finite type, given $P, Q \in \mathcal{P}(S)$, there exists a hierarchy path $\rho=\rho(P, Q):[0, n] \rightarrow \mathcal{P}(S)$ with $\rho(0)=P$, $\rho(n)=Q$. Moreover, $\rho$ is a quasi-isometric embedding with uniformly bounded constants depending only on the topological type of $S$, which has the following properties:

H1: The hierarchy $\rho$ shadows a tight $\mathcal{C}(S)$ geodesic $g_{S}$ from a multicurve $p \in P$ to a multicurve $q \in Q$, called the main geodesic of the hierarchy. That is, there is a monotonic map $v: \rho \rightarrow g_{S}$ such that for all $i, v_{i}=v(\rho(i)) \in g_{S}$ is a curve in the pants decomposition $\rho(i)$. 
H2: There is a constant $M_{1}$ such that if $Y \in \mathcal{E}(S)$ satisfies $d_{\mathcal{C}(Y)}(P, Q)>M_{1}$, then there is a maximal connected interval $I_{Y}=\left[t_{1}, t_{2}\right]$ and a tight geodesic $g_{Y}$ in $\mathcal{C}(Y)$ from a multicurve in $\rho\left(t_{1}\right)$ to a multicurve in $\rho\left(t_{2}\right)$ such that for all $t_{1} \leq t \leq t_{2}, \partial Y$ is a multicurve in $\rho(t)$, and $\left.\rho\right|_{I_{Y}}$ shadows the geodesic $g_{Y}$. Such a connected essential subsurface $Y$ is called an $M_{1}$-component domain or simply a component domain of $\rho$. By convention the entire surface $S$ is always considered a component domain.

H3: If $Y_{1} \pitchfork Y_{2}$ are two component domains of $\rho$, then there is a notion of time ordering $<_{t}$ of the domains with the property that $Y_{1}<_{t} Y_{2}$, implies $d_{Y_{2}}\left(P, \partial Y_{1}\right)<$ $M_{1}$ and $d_{Y_{1}}\left(Q, \partial Y_{2}\right)<M_{1}$. Moreover, the time ordering is independent of the choice of the hierarchy $\rho$ from $P$ to $Q$.

H4: For $Y$ a component domain with $I_{Y}=\left[t_{1}, t_{2}\right]$, let $0 \leq s \leq t_{1}, t_{2} \leq u \leq n$. Then

$$
d_{\mathcal{C}(Y)}\left(\rho(s), \rho\left(t_{1}\right)\right), d_{\mathcal{C}(Y)}\left(\rho(u), \rho\left(t_{2}\right)\right) \leq M_{1} .
$$

As a corollary of Theorem 2.9, we have the following quasi-distance formula for computing distances in $\mathcal{P}(S)$ in terms of a sum of subsurface projection distances, where the sum is over all connected essential subsurfaces above a certain threshold.

Theorem 2.10 (Quasi-distance formula [19, Theorem 6.12]) For $S=S_{g, n}$ there exists a minimal threshold $M_{2}$ depending only on the surface $S$ and quasi-isometry constants depending only on the surface $S$ and the threshold $M \geq M_{2}$ such that $d_{\mathcal{P}(S)}(P, Q)$ is quasi-isometric to $\sum_{Y \in \mathcal{E}(S)}\left\{d_{\mathcal{C}(Y)}(P, Q)\right\}_{M}$, where the threshold function $\{f(x)\}_{M}:=f(x)$ if $f(x) \geq M$, and 0 otherwise.

Note that by setting $M^{\prime}=\max \left\{10, K, M_{1}, M_{2}\right\}$ we have a single constant $M^{\prime}$, depending only on the topology of the surface $S$, which simultaneously satisfies Lemmas 2.6 and 2.8, and Theorems 2.7, 2.9 and 2.10. Throughout we will use this constant $M^{\prime}$.

Sequences of hierarchies in the pants complex give rise to ultralimits of hierarchies in the asymptotic cone of the pants complex. Specifically, given $x_{\omega}, y_{\omega} \in \mathcal{P}_{\omega}(S)$ with representatives $\left(x_{i}\right),\left(y_{i}\right)$, respectively, let $\rho_{\omega}$ be the ultralimit of the sequence of hierarchy paths $\rho_{i}$ from $x_{i}$ to $y_{i}$. Since $\rho_{i}$ are quasi-geodesics with uniform constants, $\rho_{\omega}$ is a biLipschitz quasi-geodesic in the asymptotic cone from $x_{\omega}$ to $y_{\omega}$.

\subsection{Product regions, extensions of multicurves, and regions of sublinear growth}

Given a multicurve $C \subset \mathcal{C}(S)$, by Theorem 2.10 we have a natural product region

$$
\mathcal{Q}(C) \equiv\{P \in \mathcal{P}(S) \mid C \subset P\} .
$$


Consider that an element $Q \in \mathcal{Q}(C)$ is determined by a choice of a pants decomposition of $S \backslash C$. Hence, $\mathcal{Q}(C)$ can be naturally identified with $\mathcal{P}(S \backslash C)$, which has non-trivial product structure in the event that $S \backslash C$ is a disjoint union of two or more connected essential subsurfaces. For example, given $W \in \mathcal{S E}(S), \mathcal{Q}(\partial W)$ is quasi-isometric to $\mathcal{P}(W) \times \mathcal{P}\left(W^{c}\right)$.

Given an asymptotic cone $\mathcal{P}_{\omega}(S)$ and element of the ultrapower of multicurves $\bar{C}$ we have an ultralimit

$$
\mathcal{Q}_{\omega}(\bar{C})=:\left\{x_{\omega} \in \mathcal{P}_{\omega}(S) \mid x_{\omega} \text { has a representative }\left(x_{i}^{\prime}\right) \text { with } x_{i}^{\prime} \in \mathcal{Q}\left(C_{i}\right) \omega \text {-as }\right\} .
$$

Note that unless

$$
\lim _{\omega} \frac{1}{s_{i}} d_{\mathcal{P}(S)}\left(P_{i}^{0}, \mathcal{Q}\left(C_{i}\right)\right)<\infty
$$

the ultralimit $\mathcal{Q}_{\omega}(\bar{C})$ is trivial. On the other hand, if

$$
\lim _{\omega} \frac{1}{s_{i}} d_{\mathcal{P}(S)}\left(P_{i}^{0}, \mathcal{Q}\left(C_{i}\right)\right)<\infty,
$$

then $\mathcal{Q}_{\omega}(\bar{C})$ can be naturally identified with $\mathcal{P}_{\omega}(S \backslash \bar{C})$, which has a non-trivial product structure in the event that the multicurves $C_{i} \omega$-as separate the surface $S$ into at least two disjoint connected essential subsurfaces. Recall that we always assume essential subsurfaces have complexity at least one.

Given a multicurve $C$ on a surface $S$ and a pants decomposition $X \in \mathcal{P}(S)$, we define the coarsely well-defined extension of $C$ by $X$, denoted $C\lrcorner X$, by

$$
C\lrcorner X \equiv C \cup \pi_{\mathcal{P}(S \backslash C)}(X) .
$$

More generally, for $\bar{C}$ an element of the ultrapower of multicurves satisfying

$$
\lim _{\omega} \frac{1}{s_{i}} d_{\mathcal{P}(S)}\left(P_{i}^{0}, \mathcal{Q}\left(C_{i}\right)\right)<\infty,
$$

and $x_{\omega} \in \mathcal{P}_{\omega}(S)$ we can define the extension of $\bar{C}$ by $x_{\omega}$, denoted $\left.\bar{C}\right\lrcorner x_{\omega}$, by

$$
\left.\bar{C}\lrcorner x_{\omega} \equiv \lim _{\omega}\left(C_{i}\right\lrcorner X_{i}\right) \in \mathcal{P}_{\omega}(S),
$$

where $\left(X_{i}\right)$ is any representative of $x_{\omega}$.

With the result of Theorem 2.10 in mind, [1] and later [6] developed a stratification of $\mathcal{P}_{\omega}(S)$ by considering regions of so-called sublinear growth. Specifically, given $\bar{W} \in \mathcal{E}^{\omega}(S)$ and $x_{\omega} \in \mathcal{P}_{\omega}(\bar{W})$, we define the subset of $\mathcal{P}_{\omega}(\bar{W})$ with sublinear growth from $x_{\omega}$, denoted $F_{\bar{W}, x_{\omega}}$, as

$$
F_{\bar{W}, x_{\omega}}=\left\{y_{\omega} \in \mathcal{P}_{\omega}(\bar{W}) \mid \text { for all } \bar{U} \subsetneq \bar{W}, d_{\mathcal{P}_{\omega}(\bar{U})}\left(x_{\omega}, y_{\omega}\right)=0\right\} .
$$


The following theorem organizes some properties of subsets of sublinear growth.

Theorem 2.11 [6, Theorem 3.1] With the same notation as above:

S1: $z_{\omega} \neq z_{\omega}^{\prime} \in F_{\bar{W}, x_{\omega}} \Rightarrow \lim _{\omega} d_{\mathcal{C}\left(W_{i}\right)}\left(z_{i}, z_{i}^{\prime}\right)=\infty$ for $\left(z_{i}\right),\left(z_{i}^{\prime}\right)$ any representatives of $z_{\omega}, z_{\omega}^{\prime}$, respectively. In particular, if $\gamma_{i}$ is a hierarchy between $z_{i}$ and $z_{i}^{\prime}$ shadowing a tight main geodesic $\beta_{i}$ in $\mathcal{C}\left(W_{i}\right)$ connecting any curves in the simplices $z_{i}$ and $z_{i}^{\prime}$, then $\lim _{\omega}\left|\beta_{i}\right|$ is unbounded.

S2: $\quad F_{\bar{W}, x_{\omega}} \subset \mathcal{P}_{\omega}(\bar{W})$ is an $\mathbb{R}$-tree.

S3: There is a continuous nearest point projection $\rho_{\bar{W}, x_{\omega}}: \mathcal{P}_{\omega}(\bar{W}) \rightarrow F_{\bar{W}}, x_{\omega}$, where $\rho \bar{W}, x_{\omega}$ is the identity on $F_{\bar{W}}, x_{\omega}$ and locally constant on $\mathcal{P} \omega(\bar{W}) \backslash F_{\bar{W}}, x_{\omega}$.

In [6], regions of sublinear growth are used to stratify product regions in the asymptotic cone. Specifically, for $\bar{W} \in \mathcal{E}^{\omega}(S)$ such that

$$
\lim _{\omega} \frac{1}{s_{i}} d_{\mathcal{P}(S)}\left(P^{0}, \mathcal{Q}\left(\partial W_{i}\right)\right)<\infty,
$$

and $x_{\omega} \in \mathcal{P}_{\omega}(\bar{W})$, we define the set $P_{\bar{W}, x_{\omega}} \subset \mathcal{Q}_{\omega}(\overline{\partial W})$ as

$$
P_{\bar{W}, x_{\omega}}=\left\{y_{\omega} \in \mathcal{Q}_{\omega}(\overline{\partial W}) \mid \pi_{\mathcal{P}_{\omega}(\bar{W})}\left(y_{\omega}\right) \in F_{\bar{W}, x_{\omega}}\right\} \cong \mathcal{P}_{\omega}\left(\overline{W^{c}}\right) \times F_{\bar{W}, x_{\omega}} .
$$

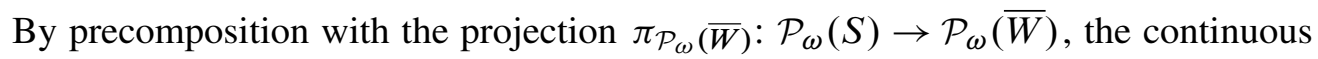
nearest point projection of property $\mathrm{S} 3$ gives rise to a continuous map

$$
\Phi_{\bar{W}, x_{\omega}}=\rho_{\bar{W}, x_{\omega}} \circ \pi_{\mathcal{P}_{\omega}(\bar{W})}: \mathcal{P}_{\omega}(S) \rightarrow F_{\bar{W}, x_{\omega}} .
$$

The following theorem regarding the above projection is an extension of Theorem 2.11.

Theorem 2.12 [6, Theorem 3.5] $\Phi_{\bar{W}, x_{\omega}}$ restricted to $P_{\bar{W}, x_{\omega}}$ is a projection onto the $F_{\bar{W}}, x_{\omega}$ factor in its natural product structure, and $\Phi_{\bar{W}}, x_{\omega}$ is locally constant on $\mathcal{P}_{\omega}(S) \backslash P_{\bar{W}}, x_{\omega}$.

The following lemma shows that the sets $F_{\bar{W}}, x_{\omega}$ can be used to study distance in $\mathcal{P}_{\omega}(S)$.

Lemma 2.13 [6, Theorem 3.6] For all $x_{\omega} \neq y_{\omega}$ in $\mathcal{P}_{\omega}(S)$, there is a $\bar{W}$ in $\mathcal{E}^{\omega}(S)$ such that

$$
\lim _{\omega} \frac{1}{s_{i}} d_{\mathcal{P}(S)}\left(P_{i}^{0}, \mathcal{Q}\left(\partial W_{i}\right)\right)<\infty
$$

with the property that $\pi_{\mathcal{P}_{\omega}(\bar{W})}\left(x_{\omega}\right) \neq \pi_{\mathcal{P}_{\omega}(\bar{W})}\left(y_{\omega}\right) \in F_{\bar{W}}, x_{\omega}$. 


\subsection{Jets}

In [5], subsets of $\mathcal{P}_{\omega}(S)$ called jets are developed. Jets are particular subsets of the asymptotic cone corresponding to sequences of geodesics in the curve complexes of connected essential subsurfaces which give rise to separation properties in $\mathcal{P}_{\omega}(S)$.

Fix $P, Q \in \mathcal{P}(S), Y \in \mathcal{E}(S)$ a connected essential subsurface, and $\sigma$ a tight geodesic in $\mathcal{C}(Y)$ from an element of $\pi_{\mathcal{C}(Y)}(P)$ to an element of $\pi_{\mathcal{C}(Y)}(Q)$. If $g=[\alpha, \beta]$ is a subsegment of $\sigma,(g, P, Q)$ is called a tight triple supported in $Y$ with ambient geodesic $\sigma$. For $(g, P, Q)$ a tight triple as above, we define the initial pants of the triple, denoted $\iota(g, P, Q) \equiv \alpha \cup \pi_{\mathcal{P}(S \backslash \alpha)}(P)$. Similarly, we define the terminal pants of the triple, denoted $\tau(g, P, Q) \equiv \beta \cup \pi_{\mathcal{P}(S \backslash \beta)}(Q)$. Then, we define the length of a tight triple supported in $Y$ by

$$
\|g\|=\|(g, P, Q)\|_{Y} \equiv d_{\mathcal{P}(Y)}(\iota(g, P, Q), \tau(g, P, Q)) .
$$

For $\bar{P}, \bar{Q} \in \mathcal{P}^{\omega}(S)$ which have non-trivial ultralimits in $\mathcal{P}_{\omega}(S)$, a Jet $\mathrm{J}$, is a quadruple of ultrapowers $(\bar{g}, \bar{Y}, \bar{P}, \bar{Q})$, where $\left(g_{i}, P, Q\right)$ are tight triples supported in $Y_{i}$. Associated to our jet J with support $\bar{Y}$ we have an initial point or basepoint of our jet $\iota(J)=\iota_{\omega}(\bar{g}, \bar{P}, \bar{Q}) \in \mathcal{P}_{\omega}(S)$ with a representative ultrapower $\iota\left(g_{i}, P, Q\right)$. Similarly, we a terminal point of our jet $\tau(J)=\tau_{\omega}(\bar{g}, \bar{P}, \bar{Q}) \in \mathcal{P}_{\omega}(S)$ with a representative ultrapower $\tau\left(g_{i}, P, Q\right)$. A jet is called macroscopic if $\iota(J) \neq \tau(J)$ and microscopic otherwise. To simplify notation, we set $\left\|\left(g_{i}, P, Q\right)\right\|_{Y_{i}}=\left\|g_{i}\right\|_{J}$. We will only consider microscopic jets.

Let J be a microscopic jet with support $\bar{Y}$ and tight geodesics $g_{i}$. Then we can consider the ultralimit $\mathcal{Q}_{\omega}(\bar{\imath} \cup \overline{\partial Y})$ which can be though of as $\iota(J) \times \mathcal{P}_{\omega}\left(\overline{Y^{c}}\right) \subset \mathcal{P}_{\omega}(S)$. Then we can define an equivalence relation on $\mathcal{P}_{\omega}(S) \backslash\left(\iota(J) \times \mathcal{P}_{\omega}\left(\overline{Y^{c}}\right)\right)$ given by

$$
x_{\omega} \sim_{J} x_{\omega}^{\prime} \Longleftrightarrow \lim _{\omega} d_{\mathcal{C}\left(Y_{i}\right)}\left(\pi_{g_{i}}\left(x_{i}\right), \pi_{g_{i}}\left(x_{i}^{\prime}\right)\right)<\infty .
$$

The following theorems regarding the existence and separation properties of microscopic jets will have application in Section 4.

Theorem 2.14 [5, Lemma 7.5] Let $a_{\omega}, b_{\omega} \in \mathcal{P}_{\omega}(S)$ with representatives $\left(a_{i}\right),\left(b_{i}\right)$ respectively. Assume that $\bar{W} \in \mathcal{E}^{\omega}(S)$ is such that $\lim _{\omega} d_{\mathcal{C}(W)}\left(a_{i}, b_{i}\right)=\infty$. Then there is a microscopic jet $J=(\bar{g}, \bar{W}, \bar{a}, \bar{b})$ such that $a_{\omega} \chi_{J} b_{\omega}$. Moreover, the subsegments $g_{i}$ can be constructed to be contained in tight $\mathcal{C}\left(W_{i}\right)$ geodesic of a hierarchy between $a_{i}$ and $b_{i}$.

Theorem 2.15 [5, Theorem 7.2] For $J$ a microscopic jet, each equivalence class under the relation $\sim_{J}$ is open. In particular, $x_{\omega}, x_{\omega}^{\prime} \in \mathcal{P}_{\omega}(S) \backslash\left(\iota(J) \times \mathcal{P}_{\omega}\left(\overline{Y^{c}}\right)\right)$, $x_{\omega} \chi_{J} x_{\omega}^{\prime} \Longrightarrow x_{\omega}$ and $x_{\omega}^{\prime}$ are separated by $\iota(J) \times \mathcal{P}_{\omega}\left(\overline{Y^{c}}\right)$. 


\section{Complex of separating multicurves}

Definition 3.1 (Separating complex) Given a surface $S$ of finite type, define the separating complex, denoted $\mathbb{S}(S)$, to have vertices corresponding to isotopy classes of separating multicurves $C \subset \mathcal{C}(S)$, that is multicurves $C$ such that at least two connected components of $S \backslash C$ are essential subsurfaces. More generally, the separating complex has $k$-cells corresponding to a sets of $k+1$ isotopy classes of separating multicurves the complement of whose union in the surface $S$ contains an essential subsurface. As usual, we will be interested in the one skeleton of $\mathbb{S}(S)$ equipped with the graph metric.

Note that in Definition 3.1 we did not require disjointness between separating multicurves corresponding to adjacent vertices. If we let $\mathbb{S}^{\prime}(S)$ denote a natural relative of our separating complex defined identically to $\mathbb{S}(S)$ in conjunction with an additional assumption of disjointness between representatives of adjacent vertices, then we have the bi-Lipschitz relation

$$
\text { for all } C, D \in \mathbb{S}(S), \quad d_{\mathbb{S}(S)}(C, D) \leq d_{\mathbb{S}^{\prime}(S)}(C, D) \leq 2 d_{\mathbb{S}(S)}(C, D) .
$$

An immediate consequence of the definition of $\mathbb{S}^{\prime}(S)$ in conjunction with Equation (3-1) is the inequality

$$
d_{\mathcal{C}(S)}(C, D) \leq d_{\mathbb{S}^{\prime}(S)}(C, D) \leq 2 d_{\mathbb{S}(S)}(C, D)
$$

Lemma 3.2 Let $C, D \in \mathbb{S}(S) . d_{\mathbb{S}(S)}(C, D) \geq 4$ implies that any connected essential subsurface of $S \backslash C$ overlaps any connected essential subsurface of $S \backslash D$.

In light of our definitions, we have the following lemma.

Lemma 3.3 Let $\bar{W}, \bar{V} \in \mathcal{S \mathcal { E } ^ { \omega }}(S)$ such that $\omega$-as $d_{\mathbb{S}(S)}\left(\partial W_{i}, \partial V_{i}\right) \geq 2$. Then

$$
\Phi_{\bar{W}, x_{\omega}}\left(\mathcal{Q}_{\omega}(\overline{\partial V})\right)=\{\mathrm{pt}\}, \quad \Phi_{\bar{V}, y_{\omega}}\left(\mathcal{Q}_{\omega}(\overline{\partial W})\right)=\{\mathrm{pt}\},
$$

where $\Phi_{\bar{W}}, x_{\omega}$ is the projection defined in Equation (2-2).

Proof Recall the definition of $\Phi_{\bar{W}, x_{\omega}}=\rho \bar{W}, x_{\omega} \circ \pi_{\mathcal{P}(\bar{W})}$. By assumption, the complement in the surface $S$ of $\partial W_{i} \cup \partial V_{i} \omega$-as does not contain an essential subsurface. Hence, it follows that $\pi_{\mathcal{P}(\bar{W})}\left(\mathcal{Q}_{\omega}(\overline{\partial V})\right)=\{\mathrm{pt}\}$, as for any $a_{\omega} \in \mathcal{Q}_{\omega}(\overline{\partial V})$ we can choose a representative $\left(a_{i}\right)$ of $a_{\omega}$ which $\omega$-as contains $\partial V_{i}$. Thus, the projection to $\mathcal{P}\left(W_{i}\right)$ is coarsely entirely determined by the projection of the curve $\partial V_{i}$. 


\subsection{Connected components of $\mathbb{S}\left(S_{2,1}\right)$ and point pushing}

Recall that the connected components of $\mathbb{S}^{\prime}(S)$ and $\mathbb{S}(S)$ are equivalent. By topological considerations, $\mathbb{S}^{\prime}\left(S_{2,1}\right)$ consists of separating curves or disjoint pairs thereof. Hence, vertices of $\mathbb{S}^{\prime}\left(S_{2,1}\right)$ and simplices of $\mathcal{C}_{\text {sep }}\left(S_{2,1}\right)$ are in correspondence. Moreover, vertices in $\mathbb{S}^{\prime}\left(S_{2,1}\right)$ are adjacent if and only if the corresponding simplices are adjacent in $\mathcal{C}_{\text {sep }}\left(S_{2,1}\right)$. Thus, the connected components of $\mathbb{S}^{\prime}\left(S_{2,1}\right)$, or equivalently $\mathbb{S}\left(S_{2,1}\right)$, are precisely the connected components of $\mathcal{C}_{\text {sep }}\left(S_{2,1}\right)$.

Let $\pi_{\mathcal{C}\left(S_{2,0}\right)}: \mathcal{C}\left(S_{2,1}\right) \rightarrow \mathcal{C}\left(S_{2,0}\right)$ be the projection given by forgetting about the boundary component. Up to homeomorphism there is only one separating curve on the surfaces $S_{2,1}$ and $S_{2,0}$. In fact under the projection $\pi_{\mathcal{C}\left(S_{2,0}\right)}$ the image of a separating curve is a separating curve, and similarly the preimage of a separating curve is a union of separating curves. In other words, the map $\pi_{\mathcal{C}\left(S_{2,0}\right)}$ has a natural well-defined surjective restriction $\mathcal{C}_{\text {sep }}\left(S_{2,1}\right) \rightarrow \mathcal{C}_{\text {sep }}\left(S_{2,0}\right)$. Moreover, the fibers of $\pi_{\mathcal{C}_{\text {sep }}}$ are connected [20].

Lemma 3.4 The fibers of $\pi_{\mathcal{C}_{\text {sep }}}$ coincide with the connected components of $\mathcal{C}_{\text {sep }}\left(S_{2,1}\right)$. In particular, since there are infinitely many curves in the range, $\mathcal{C}_{\text {sep }}\left(S_{2,0}\right)$, it follows that there are infinitely many fibers, and hence infinitely many connected components of $\mathcal{C}_{\text {sep }}\left(S_{2,1}\right)$.

Proof Since the fibers of $\pi_{\mathcal{C}_{\text {sep }}}$ are connected, to prove the lemma it suffices to show that any two curves $\alpha, \beta$ which can be connected in $\mathcal{C}_{\text {sep }}\left(S_{2,1}\right)$ must satisfy $\pi_{\mathcal{C}}(\alpha)=\pi_{\mathcal{C}}(\beta)$. Without loss of generality we can assume that $\alpha \cap \beta=\varnothing$. Ignoring the boundary component, we have disjoint representatives of $\pi_{\mathcal{C}}(\alpha)$, and $\pi_{\mathcal{C}}(\beta)$. However, there are no distinct isotopy classes of separating curves in $S_{2,0} \Rightarrow \pi_{\mathcal{C}}(\alpha)=\pi_{\mathcal{C}}(\beta)$.

Lemma 3.5 The point pushing subgroup Push $\subset \mathcal{M C G}\left(S_{2,1}\right)$ preserves the connected components of $\mathcal{C}_{\text {sep }}\left(S_{2,1}\right)$. Similarly, Push $\subset \mathcal{M C G}\left(S_{2,1}\right)$ preserves the fibers of the projection $\pi_{\mathcal{P}}: \mathcal{P}\left(S_{2,1}\right) \rightarrow \mathcal{P}\left(S_{2,0}\right)$.

Since there exist pseudo-Anosov point pushing maps [16] and because pseudo-Anosov axes have infinite diameter in $\mathcal{C}(S)$ [18], which in particular ensures that the axes have infinite diameter in $\mathcal{C}_{\text {sep }}(S)$, by Lemma 3.5 it follows that the connected components of $\mathcal{C}_{\text {sep }}\left(S_{2,1}\right)$ have infinite diameter. Putting together Lemmas 3.4 and 3.5, we have the following corollary which uniquely characterizes the surface $S_{2,1}$ and which is the underlying reason for the unique phenomenon regarding the thickness of $\mathcal{T}\left(S_{2,1}\right)$ studied in Section 5.

Corollary 3.6 $\mathcal{C}_{\text {sep }}\left(S_{2,1}\right)$, and similarly $\mathbb{S}\left(S_{2,1}\right)$, has infinitely many connected components, each with infinite diameter. 


\section{2 $\mathbb{S}_{\omega}(S)$, the ultralimit of $\mathbb{S}(S)$}

Throughout this section we assume a fixed asymptotic cone $\mathcal{P}_{\omega}(S)$, and consider the ultralimit of $\mathbb{S}(S)$, which we denote $\mathbb{S}_{\omega}(S)$.

Definition 3.7 $\left(\mathbb{S}_{\omega}(S)\right)$ Given a surface $S$ of finite type, define $\mathbb{S}_{\omega}(S)$ to have vertices corresponding to $\bar{C} \in \mathbb{S}(S)^{\omega}$ such that

$$
\lim _{\omega} \frac{1}{s_{i}} d_{\mathcal{P}(S)}\left(P_{i}^{0}, \mathcal{Q}\left(C_{i}\right)\right)<\infty .
$$

Equivalently, vertices in $\mathbb{S}_{\omega}(S)$ correspond to natural product regions $\mathcal{Q}_{\omega}(\bar{C}) \subset \mathcal{P}_{\omega}(S)$. By abuse of notation, we will sometimes interchange between these two equivalent descriptions of vertices in $\mathbb{S}_{\omega}(S)$. Furthermore, define $\mathbb{S}_{\omega}(S)$ to have an edge between vertices $\mathcal{Q}_{\omega}(\bar{C})$ and $\mathcal{Q}_{\omega}(\bar{D})$ if in the asymptotic cone $\mathcal{Q}_{\omega}(\overline{C\lrcorner D})=\mathcal{Q}_{\omega}(\overline{D\lrcorner C})$, and moreover $\omega$-as the complement $S \backslash\left\{C_{i}, D_{i}\right\}$ contains an essential subsurface $Y_{i}$.

Given our definition of $\mathbb{S}_{\omega}(S)$, we can define a related $[0, \infty]$-valued pseudometric on the asymptotic cone which gives information about the natural product structures connecting points in the asymptotic cone. Specifically, define

$$
d_{\mathbb{S}_{\omega}(S)}\left(a_{\omega}, b_{\omega}\right) \equiv \inf _{\bar{A}, \bar{B}} d_{\mathbb{S}_{\omega}(S)}(\bar{A}, \bar{B}),
$$

where the infimum is taken over all pairs $\bar{A}, \bar{B}$ in the vertex set of $\mathbb{S}_{\omega}(S)$ having the property that $a_{\omega} \in \mathcal{Q}_{\omega}(\bar{A})$ and $b_{\omega} \in \mathcal{Q}_{\omega}(\bar{B})$.

This definition is well-defined, as given any pants decompositions $P \in \mathcal{P}(S)$ there is a bound $D(S)$ depending only on the topological type of the surface $S$, such that there is a pants decomposition $P^{\prime} \in \mathcal{P}(S)$ containing a separating curve and $d_{\mathcal{P}(S)}\left(P, P^{\prime}\right) \leq D(S)$. In particular, given any element of the asymptotic cone $a_{\omega}$ with any representative $\left(A_{i}\right)$ there exists an alternative representative $\left(A_{i}^{\prime}\right)$, with $A_{i}^{\prime}$ containing a separating curve, thus making it clear that $a_{\omega}$ lies in some natural product region of the asymptotic cone. The following theorem whose proof is a straightforward application of definitions ensures appropriate compatibility of $\mathbb{S}(S)$ and $\mathbb{S}_{\omega}(S)$.

Theorem 3.8 Let $\bar{C}, \bar{D}$ be vertices in $\mathbb{S}_{\omega}(S)$. Then $d_{\mathbb{S}_{\omega}(S)}(\bar{C}, \bar{D})$ is quasi-isometric to $\lim _{\omega} d_{\mathbb{S}(S)}\left(C_{i}, D_{i}\right)$. Moreover, when $d_{\mathbb{S}_{\omega}(S)}(\bar{C}, \bar{D})$ is finite yet non-trivial, for each of the finite number of natural product regions $\mathcal{Q}_{\omega}(\bar{A}) \subset \mathcal{P}_{\omega}(S)$ traveled through in the path between $\mathcal{Q}_{\omega}(\bar{C})$ and $\mathcal{Q}_{\omega}(\bar{D})$, the separating curve $A_{i}$ is $\omega$-as in the same connected components as the finite $\mathbb{S}(S)$ geodesic from $C_{i}$ to $D_{i}$. 
The bi-Lipschitz relation in Theorem 3.8 guarantees that one of the terms is infinite if and only if the other term is infinite. It should be stressed that the term $\lim _{\omega} d_{\mathbb{S}(S)}\left(C_{i}, D_{i}\right)$ can be infinite due to two different reasons. It is possible that $\omega-$ as $C_{i}$ and $D_{i}$ are connected in $\mathbb{S}(S)$ however their distances are unbounded. On the other hand, for small enough complexity surfaces, it is possible that $\omega$-as $C_{i}$ and $D_{i}$ are in different connected components of $\mathbb{S}(S)$. This distinction will be crucial in Section 4.

\section{Asymptotic cone of Teichmüller space}

\subsection{Structurally integral corners}

Informally, a structurally integral corner entails the joining of two particular natural product regions in the asymptotic cone of the pants complex at a "corner" such that the removal of the corner joining the regions separates the two product regions from each other. More formally, fixing some ultrafilter $\omega$, we have the following definition:

Definition 4.1 (Structurally integral corner) Let $\bar{\alpha} \neq \bar{\beta} \in \mathbb{S}^{\omega}$ be such that the following conditions hold:

(1) $\omega$-as $\alpha_{i}$ and $\beta_{i}$ are in different connected components of $\mathbb{S}(S)$. In particular, it follows that $\lim _{\omega} d_{\mathbb{S}(S)}\left(\alpha_{i}, \beta_{i}\right)=\infty$ and $\left.\left.\alpha_{i}\right\lrcorner \beta_{i}, \beta_{i}\right\lrcorner \alpha_{i} \in \mathcal{P}(S)$.

(2) $\left.\left.\lim _{\omega} d_{\mathcal{P}(S)}\left(\alpha_{i}\right\lrcorner \beta_{i}, \beta_{i}\right\lrcorner \alpha_{i}\right)$ is bounded. In particular, for any $\bar{Y} \in \mathcal{E}^{\omega}(S)$, the limit

$$
\left.\left.\lim _{\omega} d_{\mathcal{C}\left(Y_{i}\right)}\left(\alpha_{i}\right\lrcorner \beta_{i}, \beta_{i}\right\lrcorner \alpha_{i}\right) \text { is bounded. }
$$

In this setting we call the point $(\alpha\lrcorner \beta)^{\omega}$ (or equivalently the point $\left.(\beta\lrcorner \alpha\right)^{\omega}$ ) a structurally integral corner, and denote it by $\bar{\alpha} C_{\bar{\beta}}$.

Notice that by Definition 4.1(1), structurally integral corners can only exist for surfaces $S$ with disconnected separating complexes, or equivalently for surfaces with $|\chi(S)| \leq 4$; see [21, Theorem 3.1.1].

After descending from elements of ultrapowers to elements of the asymptotic cone, the structurally integral corners $(\alpha\lrcorner \beta)_{\omega}$ and $\left.(\beta\lrcorner \alpha\right)_{\omega}$ will be identified and moreover, this point will serve as a cut-point between the natural product regions $\mathcal{Q}_{\omega}(\bar{\alpha})$ and $\mathcal{Q}_{\omega}(\bar{\beta})$. We must assume that our cone $\mathcal{P}_{\omega}(S)$ contains the corner $\left.(\alpha\lrcorner \beta\right)_{\omega}$, or equivalently we must assume $\left.\lim _{\omega} \frac{1}{s_{i}} d_{\mathcal{P}(S)}\left(P_{i}^{0}, \alpha_{i}\right\lrcorner \beta_{i}\right)<\infty$. 
Example 4.2 (A structurally integral corner in $\mathcal{P} \omega\left(S_{2,1}\right)$ ) Let $\alpha_{i}, \beta_{i} \in \mathcal{C}_{\text {sep }}\left(S_{2,1}\right)$ be such that

$$
\lim _{\omega} \frac{1}{s_{i}} d_{\mathcal{P}(S)}\left(P_{i}^{0}, \mathcal{Q}\left(\alpha_{i}\right)\right)<\infty, \quad \lim _{\omega} \frac{1}{s_{i}} d_{\mathcal{P}(S)}\left(P_{i}^{0}, \mathcal{Q}\left(\beta_{i}\right)\right)<\infty
$$

Moreover, assume that $\omega$-as (i) the intersection number $i\left(\alpha_{i}, \beta_{i}\right)$ is bounded, and (ii) $\alpha_{i}, \beta_{i}$ are in different connected components of $\mathcal{C}_{\text {sep }}(S)$. In this case $\bar{\alpha} C_{\bar{\beta}}$ is a structurally integral corner in $\mathcal{P}_{\omega}\left(S_{2,1}\right)$. The only non-trivial point to note is that the bound on the intersection number between $\alpha_{i}$ and $\beta_{i}$ guarantees condition (2) of Definition 4.1.

Given the notion of a structurally integral corner, we will now introduce a relation $\sim_{\bar{\alpha}, \bar{\beta}}$ on $\mathcal{P}^{\omega}(S)$ which descends to an equivalence relation on $\mathcal{P}_{\omega}(S) \backslash \bar{\alpha} C_{\bar{\beta}}$. Moreover, each equivalence class is open. In particular, it will follow that in the asymptotic cone, $\mathcal{P}_{\omega}(S)$, the corner $\bar{\alpha} C_{\bar{\beta}}$ is a cut-point between points of $\mathcal{P}_{\omega}(S) \backslash \bar{\alpha} C_{\bar{\beta}}$ which are in different equivalence classes under the relation $\sim_{\bar{\alpha}, \bar{\beta}}$. We begin with the following definition of a relation $\sim_{\bar{\alpha}, \bar{\beta}}$ on $\mathcal{P}^{\omega}(S)$.

Definition 4.3 Let $\bar{\alpha} C_{\bar{\beta}}$ be a structurally integral corner. Then we have an equivalence relation $\sim \bar{\alpha}, \bar{\beta}$ on $\mathcal{P}^{\omega}(S)$ given by saying $\bar{P} \sim \bar{\alpha}, \bar{\beta} \bar{Q}$ if and only if $\bar{P}$ and $\bar{Q}$ fall into the same case under the following trichotomy. Namely, given $\bar{P}$ :

(1) $\bar{P}$ is in case one if there exists $\overline{W_{\alpha}} \in \mathcal{S E}^{\omega}(S)$ such that the following two conditions hold:

(i) $\lim _{\omega} d_{\mathbb{S}(S)}\left(\alpha_{i}, \partial W_{\alpha, i}\right)$ is bounded.

(ii) $\lim _{\omega} d_{\mathcal{C}\left(W_{\alpha, i}\right)}\left(P_{i}, \beta_{i}\right)=\infty$.

(2) $\bar{P}$ is in case two if there exists $\overline{W_{\beta}} \in \mathcal{S E}^{\omega}(S)$ such that the following two conditions hold:

(i) $\lim _{\omega} d_{\mathbb{S}(S)}\left(\beta_{i}, \partial W_{\beta, i}\right)$ is bounded.

(ii) $\lim _{\omega} d_{\mathcal{C}\left(W_{\beta, i}\right)}\left(P_{i}, \alpha_{i}\right)=\infty$.

(3) $\bar{P}$ is in case three if neither the conditions of case one nor case two apply to $\bar{P}$.

As a first order of business, the following lemma guarantees the mutual exclusivity of the three cases in the definition of $\sim_{\bar{\alpha}, \bar{\beta}}$, thus ensuring that the equivalence relation of Definition 4.3 is well-defined.

Lemma 4.4 Let $\bar{P} \in \mathcal{P}^{\omega}(S)$. Then $\bar{P}$ falls into one and only one of the three cases in the trichotomy of Definition 4.3. 
Proof It suffices to show that $\bar{P}$ cannot simultaneously be in cases one and two. Assume not, that is, assume there are elements $\overline{W_{\alpha}}, \overline{W_{\beta}} \in \mathcal{S E}^{\omega}(S)$ such that

$$
\lim _{\omega} d_{\mathbb{S}(S)}\left(\alpha_{i}, \partial W_{\alpha, i}\right) \quad \text { and } \quad \lim _{\omega} d_{\mathbb{S}(S)}\left(\beta_{i}, \partial W_{\beta, i}\right)
$$

are bounded (and by Equation (3-1) similarly for $\mathbb{S}^{\prime}(S)$ ), while

$$
\lim _{\omega} d_{\mathcal{C}\left(W_{\alpha, i}\right)}\left(P_{i}, \beta_{i}\right) \quad \text { and } \quad \lim _{\omega} d_{\mathcal{C}\left(W_{\beta, i}\right)}\left(P_{i}, \alpha_{i}\right)
$$

are unbounded.

Since $\bar{\alpha} C_{\bar{\beta}}$ is a structurally integral corner, in particular, we have that $\lim _{\omega} d_{\mathbb{S}(S)}\left(\alpha_{i}, \beta_{i}\right)$ is unbounded, and consequently by our assumptions, $\lim _{\omega} d_{\mathbb{S}(S)}\left(\partial W_{\alpha, i}, \partial W_{\beta, i}\right)$ is unbounded as well. Lemma 3.2 then guarantees that $\overline{W_{\alpha}} \pitchfork \overline{W_{\beta}}$.

By Lemma 2.6 if $Y_{i} \in \mathcal{E}(S) \omega$-as intersects every separating multicurve in the bounded path of disjoint separating multicurves in $\mathbb{S}^{\prime}(S)$ connecting $\beta_{i}$ and $\partial W_{\beta, i}$, then

$$
\lim _{\omega} d_{\mathcal{C}\left(Y_{i}\right)}\left(\beta_{i}, \partial W_{\beta, i}\right)
$$

is bounded as well. In particular, since the distance in $\mathbb{S}^{\prime}(S)$ between $\partial W_{\alpha, i}$ and the bounded path connecting $\beta_{i}$ and $\partial W_{\beta, i}$, is unbounded, Lemma 3.2 implies that $\omega$-as $\partial W_{\alpha, i}$ intersects every separating multicurve in the bounded path of separating multicurves in $\mathbb{S}^{\prime}(S)$ connecting $\beta_{i}$ and $\partial W_{\beta, i}$. Hence, $\lim _{\omega} d_{\mathcal{C}\left(W_{\alpha, i}\right)}\left(\beta_{i}, \partial W_{\beta, i}\right)$ is bounded. Similarly, $\lim _{\omega} d_{\mathcal{C}\left(W_{\beta, i}\right)}\left(\alpha_{i}, \partial W_{\alpha, i}\right)$ is bounded. In conjunction with our assumptions, it follows that $\lim _{\omega} d_{\mathcal{C}\left(W_{\alpha, i}\right)}\left(P_{i}, \partial W_{\beta, i}\right)$ and $\lim _{\omega} d_{\mathcal{C}\left(W_{\beta, i}\right)}\left(P_{i}, \partial W_{\alpha, i}\right)$ are unbounded. Since $\overline{W_{\alpha}} \pitchfork \overline{W_{\beta}}$, this contradicts Lemma 2.8 .

Having proven that the relation $\sim \bar{\alpha}, \bar{\beta}$ is well-defined, we will now prove that the relation in fact descends to an equivalence relation on $\mathcal{P}_{\omega}(S) \backslash \bar{\alpha} C_{\bar{\beta}}$.

Theorem 4.5 The relation $\sim \bar{\alpha}, \bar{\beta}$ descends to an equivalence relation on $\mathcal{P}_{\omega}(S) \backslash \bar{\alpha} C_{\bar{\beta}}$. Moreover, each equivalence class is open.

The proof of Theorem 4.5 will follow from the following technical lemma.

Lemma 4.6 There is a constant $C \geq 0$ such that for $\bar{\alpha} C_{\bar{\beta}}$ a structurally integral corner if $\bar{P}, \bar{Q}$ are sequences representing points $P_{\omega}, Q_{\omega} \in \mathcal{P}_{\omega}(S)$, and if $\bar{P} \chi_{\bar{\alpha}, \bar{\beta}} \bar{Q}$. Then

$$
d_{\mathcal{P}_{\omega}(S)}\left(P_{\omega}, Q_{\omega}\right) \geq C d_{\mathcal{P}_{\omega}(S)}\left(P_{\omega, \bar{\alpha}} C_{\bar{\beta}}\right) .
$$


Proof of Theorem 4.5 Assume that $\bar{P}$ and $\bar{Q}$ are representatives of the same point of the asymptotic cone. Then by Lemma 4.6 either $\bar{P} \sim \bar{\alpha}, \bar{\beta} \bar{Q}$ or in the asymptotic cone, $P_{\omega}={ }_{\alpha} C_{\bar{\beta}}$. Hence, the relation $\sim \bar{\alpha}, \bar{\beta}$ descends to a relation on $\mathcal{P}_{\omega}(S) \backslash \bar{\alpha} C_{\bar{\beta}}$ which is reflexive. Furthermore, since by definition it is immediate that $\sim_{\bar{\alpha}, \bar{\beta}}$ is symmetric and transitive, it follows that $\sim \bar{\alpha}, \bar{\beta}$ descends to an equivalence relation on $\mathcal{P}_{\omega}(S) \backslash \bar{\alpha} C_{\bar{\beta}}$. Lemma 4.6 implies that any point $P_{\omega} \in \mathcal{P}_{\omega}(S) \backslash{ }_{\bar{\alpha}} C_{\bar{\beta}}$ has an open neighborhood consisting entirely of points which are in the same equivalence class. Hence, the equivalence classes are open.

Proof of Lemma 4.6 $\left.P_{i}, Q_{i}, \alpha_{i}\right\lrcorner \beta_{i}$ are pants decompositions of a surface and hence have non-trivial subsurface projection to any essential subsurface. For any $Y \in \mathcal{E}(S)$, let $\sigma_{i}^{Y}$ be a $\mathcal{C}(Y)$ geodesic from $P_{i}$ to $Q_{i}$. Moreover, let $\left.\pi_{\sigma_{i}^{Y}}\left(\alpha_{i}\right\lrcorner \beta_{i}\right)$ be the nearest point projection of $\left.\pi_{\mathcal{C}(Y)}\left(\alpha_{i}\right\lrcorner \beta_{i}\right)$ onto the geodesic $\sigma_{i}^{Y}$. By definition, for all $Y \in \mathcal{E}(S)$ we have

$$
\left.d_{\mathcal{C}(Y)}\left(P_{i}, Q_{i}\right) \geq d_{\mathcal{C}(Y)}\left(P_{i}, \pi_{\sigma_{i}^{Y}}\left(\alpha_{i}\right\lrcorner \beta_{i}\right)\right) .
$$

In order to complete the proof we will show that there is a uniform constant $k$ such that for all $Y \in \mathcal{E}(S)$,

$$
\left.\left.d_{\mathcal{C}(Y)}\left(\alpha_{i}\right\lrcorner \beta_{i}, \pi_{\sigma_{i}^{Y}}\left(\alpha_{i}\right\lrcorner \beta_{i}\right)\right)<k .
$$

Combining Equations (4-1) and (4-2), for all $Y \in \mathcal{E}(S)$, we have

$$
\left.d_{\mathcal{C}(Y)}\left(P_{i}, Q_{i}\right) \geq d_{\mathcal{C}(Y)}\left(P_{i}, \alpha_{i}\right\lrcorner \beta_{i}\right)-k .
$$

In particular, by Theorem 2.10, in the asymptotic cone we have the following inequality thus completing the proof:

$$
d_{\mathcal{P}_{\omega}(S)}\left(P_{\omega}, Q_{\omega}\right) \geq C d_{\mathcal{P}_{\omega}(S)}\left(P_{\omega, \omega}\right) .
$$

By condition (2) in the definition of a structurally integral corner $\bar{\alpha} C_{\bar{\beta}}$ it follows that $\left.\left.\lim _{\omega} \operatorname{diam}_{\mathcal{C}(Y)}\left(\left\{\alpha_{i}, \beta_{i}, \alpha_{i}\right\lrcorner \beta_{i}, \beta_{i}\right\lrcorner \alpha_{i}\right\}\right)$ is bounded, and hence, in place of (4-2) it suffices to show that $\lim _{\omega} d_{\mathcal{C}\left(Y_{i}\right)}\left(\sigma_{i}^{Y_{i}},\left\{\alpha_{i}, \beta_{i}\right\}\right)$ is bounded.

By assumption $\bar{P}$ and $\bar{Q}$ are in different equivalence classes, and hence by definition $\bar{P}$ and $\bar{Q}$ fall into different cases in Definition 4.3. By symmetry of the cases, without loss of generality we can assume that $\bar{P}$ is in case one of Definition 4.3, while $\bar{Q}$ is not. Namely, there is a $\overline{W_{\alpha}} \in \mathcal{S E}^{\omega}(S)$ such that $\lim _{\omega} d_{\mathbb{S}(S)}\left(\alpha_{i}, \partial W_{\alpha, i}\right)$ is bounded, while $\lim _{\omega} d_{\mathcal{C}\left(W_{\alpha, i}\right)}\left(P_{i}, \beta_{i}\right)=\infty$. Furthermore, for any element $\bar{U} \in \mathcal{S E}^{\omega}(S)$ such that $\lim _{\omega} d_{\mathbb{S}(S)}\left(\alpha_{i}, \partial U_{i}\right)$ is bounded, perforce $\lim _{\omega} d_{\mathcal{C}\left(U_{i}\right)}\left(Q_{i}, \beta_{i}\right)$ is also bounded. By Equation (3-1) the same statements hold for $\mathbb{S}^{\prime}(S)$. 
We proceed by considering cases for the relationship between $\bar{Y}$ and $\overline{W_{\alpha}}$ where $\bar{Y}$ is an arbitrary element of the ultrapower of connected essential subsurfaces. By Lemma 2.1 since there are only a finite number of possibilities for the relationship between two essential subsurfaces - identical, nested, overlapping, and disjoint - the same finitely many possibilities for the relationship between $\bar{Y}$ and $\overline{W_{\alpha}}$. In each case we will show $\lim _{\omega} d_{\mathcal{C}\left(Y_{i}\right)}\left(\sigma_{i}^{Y_{i}},\left\{\alpha_{i}, \beta_{i}\right\}\right)$ is bounded, thus completing the proof of the lemma.

Case 1 Here either $\bar{Y} \subset \overline{W_{\alpha}}$ or $\bar{Y} \cap \overline{W_{\alpha}}=\varnothing$. In either case, $\omega$-as $d_{\mathbb{S}(S)}\left(\partial W_{\alpha, i}, \partial Y_{i}\right) \leq$ 1 and hence by our assumptions $\lim _{\omega} d_{\mathbb{S}(S)}\left(\alpha_{i}, \partial Y_{i}\right)$ is bounded. Since $\bar{Q}$ is not in case one of the equivalence relation $\sim \bar{\alpha}, \bar{\beta}$, it follows that $\lim _{\omega} d_{\mathcal{C}\left(Y_{i}\right)}\left(Q_{i}, \beta_{i}\right)$ is bounded. In particular, this implies that $\lim _{\omega} d_{\mathcal{C}\left(Y_{i}\right)}\left(\sigma_{i}^{Y_{i}},\left\{\alpha_{i}, \beta_{i}\right\}\right)$ is bounded, completing this case.

Case 2 Here $\overline{W_{\alpha}} \subset \bar{Y}$ and $\lim _{\omega} d_{\mathcal{C}\left(Y_{i}\right)}\left(\partial W_{\alpha, i},\left\{\alpha_{i}, \beta_{i}\right\}\right)$ is bounded. By our assumptions,

$$
\lim _{\omega} d_{\mathcal{C}\left(W_{\alpha, i}\right)}\left(P_{i}, \beta_{i}\right)=\infty,
$$

while $\lim _{\omega} d_{\mathcal{C}\left(W_{\alpha, i}\right)}\left(Q_{i}, \beta_{i}\right)$ is bounded. In particular, $\lim _{\omega} d_{\mathcal{C}\left(W_{\alpha, i}\right)}\left(P_{i}, Q_{i}\right)=\infty$. Then $\omega$-as $d_{\mathcal{C}\left(Y_{i}\right)}\left(\partial W_{\alpha, i}, \sigma_{i}^{Y_{i}}\right) \leq 1$. If not, then Theorem 2.7 would imply that $\omega-$ as $d_{\mathcal{C}\left(W_{\alpha, i}\right)}\left(P_{i}, Q_{i}\right)$ is uniformly bounded which is a contradiction. However, the assumption of the case that $\lim _{\omega} d_{\mathcal{C}\left(Y_{i}\right)}\left(\partial W_{\alpha, i},\left\{\alpha_{i}, \beta_{i}\right\}\right)$ is bounded then implies that $\lim _{\omega} d_{\mathcal{C}\left(Y_{i}\right)}\left(\left\{\alpha_{i}, \beta_{i}\right\}, \sigma_{i}^{Y_{i}}\right)$ is bounded, thus completing this case.

Case 3 Here $\bar{Y} \pitchfork \overline{W_{\alpha}}$ and $\lim _{\omega} d_{\mathcal{C}\left(Y_{i}\right)}\left(\partial W_{\alpha, i},\left\{\alpha_{i}, \beta_{i}\right\}\right)$ is bounded. As in Case 2, by our assumptions $\lim _{\omega} d_{\mathcal{C}\left(W_{\alpha, i}\right)}\left(P_{i}, \beta_{i}\right)=\infty$, while $\lim _{\omega} d_{\mathcal{C}\left(W_{\alpha, i}\right)}\left(Q_{i}, \beta_{i}\right)$ is bounded. In particular,

$$
\lim _{\omega} d_{\mathcal{C}\left(W_{\alpha, i}\right)}\left(P_{i}, Q_{i}\right)=\infty
$$

Since $\omega$-as $W_{\alpha, i} \pitchfork Y_{i}$, it follows that $\lim _{\omega} d_{\mathcal{C}\left(Y_{i}\right)}\left(\partial W_{\alpha, i},\left\{P_{i}, Q_{i}\right\}\right)$ is uniformly bounded. If not, then Lemma 2.8 implies that $d_{\mathcal{C}\left(W_{\alpha, i}\right)}\left(P_{i}, Q_{i}\right)$ is uniformly bounded which is a contradiction. However, the assumption of the case that

$$
\lim _{\omega} d_{\mathcal{C}\left(Y_{i}\right)}\left(\partial W_{\alpha, i},\left\{\alpha_{i}, \beta_{i}\right\}\right)
$$

is bounded then implies that $\lim _{\omega} d_{\mathcal{C}\left(Y_{i}\right)}\left(\left\{\alpha_{i}, \beta_{i}\right\},\left\{P_{i}, Q_{i}\right\}\right)$ is bounded. Since $\sigma_{i}^{Y_{i}}$ is $\mathcal{C}\left(Y_{i}\right)$ geodesic between $P_{i}$ and $Q_{i}$, it follows that $\lim _{\omega} d_{\mathcal{C}\left(Y_{i}\right)}\left(\left\{\alpha_{i}, \beta_{i}\right\}, \sigma_{i}^{Y_{i}}\right)$ is bounded, thus completing this case.

Case 4 Here either $\overline{W_{\alpha}} \subset \bar{Y}$ or $\bar{Y} \pitchfork \overline{W_{\alpha}}$; in both cases, $\lim _{\omega} d_{\mathcal{C}\left(Y_{i}\right)}\left(\partial W_{\alpha, i},\left\{\alpha_{i}, \beta_{i}\right\}\right)$ is unbounded. Since $\lim _{\omega} d_{\mathbb{S}^{\prime}(S)}\left(\alpha_{i}, \partial W_{\alpha, i}\right)$ is bounded, it follows that there is a bounded path of connected multicurves in the curve complex $\mathcal{C}(S)$ from $\alpha_{i}$ to $\partial W_{\alpha, i}$ such that each multicurve is a separating multicurve. Call this path $\rho_{i}$. On the other 
hand, the assumption of the case is that $\lim _{\omega} d_{\mathcal{C}\left(Y_{i}\right)}\left(\partial W_{\alpha, i},\left\{\alpha_{i}, \beta_{i}\right\}\right)=\infty$. Putting things together, by Lemma 2.6 it follows $\omega$-as $Y_{i}$ is disjoint from some vertex in $\rho_{i}$. By construction, it follows that $\partial Y_{i} \in \mathbb{S}(S)$, and in fact $\lim _{\omega} d_{\mathbb{S}(S)}\left(\alpha_{i}, \partial Y_{i}\right)$ is bounded. Since $\bar{Q}$ is not in case one of the equivalence relation $\sim \bar{\alpha}, \bar{\beta}$, it follows that $\lim _{\omega} d_{\mathcal{C}\left(Y_{i}\right)}\left(Q_{i}, \beta_{i}\right)$ is bounded. It follows that $\lim _{\omega} d_{\mathcal{C}\left(Y_{i}\right)}\left(\left\{\alpha_{i}, \beta_{i}\right\}, \sigma_{i}^{Y_{i}}\right)$ is bounded. This completes the proof of the final case thereby completing the proof of the lemma.

As an immediate corollary of Theorem 4.5 we have the following useful separation property of structurally integral corners in the asymptotic cone. This separation property should be compared with the separation property of microscopic jets recorded in Theorem 2.15.

Corollary 4.7 Let ${ }_{\bar{\alpha}} C_{\bar{\beta}}$ be a structurally integral corner, and let $x_{\omega}, x_{\omega}^{\prime} \in \mathcal{P}_{\omega}(S) \backslash{ }_{\bar{\alpha}} C_{\bar{\beta}}$ be points in the asymptotic cone such that $x_{\omega} \chi_{\bar{\alpha}, \bar{\beta}} x_{\omega}^{\prime}$. Then $x_{\omega}$ and $x_{\omega}^{\prime}$ are separated by the corner $\bar{\alpha} C_{\bar{\beta}}$.

Theorem 4.8 Let $S=S_{g, n}$ and let $\mathcal{P}_{\omega}(S)$ be any asymptotic cone of $\mathcal{P}(S)$. Then for all $a_{\omega}, b_{\omega} \in \mathcal{P}_{\omega}(S)$, the following are equivalent:

(1) No point separates $a_{\omega}$ and $b_{\omega}$, or equivalently $a_{\omega}$ and $b_{\omega}$ are in the same canonical finest piece, and

(2) In any neighborhood of $a_{\omega}, b_{\omega}$, respectively, there exist $a_{\omega}^{\prime}, b_{\omega}^{\prime}$, with representative sequences $\left(a_{i}^{\prime}\right),\left(b_{i}^{\prime}\right)$, such that $\lim _{\omega} d_{\mathbb{S}(S)}\left(a_{i}^{\prime}, b_{i}^{\prime}\right)<\infty$.

Proof (2) $\Longrightarrow$ (1) Property (2) implies that $a_{\omega}, b_{\omega}$ are limit points of sequences in the asymptotic cone which have finite $\mathbb{S}_{\omega}(S)$ distance. Since the canonically defined finest pieces are closed sets [14], it suffices to show that points in the asymptotic cone with finite $\mathbb{S}_{\omega}(S)$ distance cannot be separated by a point. Specifically, assume we have a chain of natural product regions $\mathcal{Q}_{\omega}\left(\overline{\gamma_{0}}\right), \ldots, \mathcal{Q}_{\omega}\left(\overline{\gamma_{K}}\right)$ in the asymptotic cone $\mathcal{P}_{\omega}(S)$ such that $a_{\omega}^{\prime} \in \mathcal{Q}_{\omega}\left(\overline{\gamma_{0}}\right), b_{\omega}^{\prime} \in \mathcal{Q}_{\omega}\left(\overline{\gamma_{K}}\right)$, and for all $j \in\{0, \ldots, K-1\}$ $\mathcal{Q}_{\omega}\left(\overline{\gamma_{j}}\right) \cap \mathcal{Q}_{\omega}\left(\overline{\gamma_{j+1}}\right)$ has infinite diameter intersection. Clearly, each product region cannot be separated by a point. Furthermore, by assumption each product region cannot be separated from its neighbor by a point. It follows that $a_{\omega}^{\prime}$ and $b_{\omega}^{\prime}$ cannot be separated by a point, thus completing the proof of (2) $\Longrightarrow(1)$.

(1) $\Longrightarrow$ (2) We will prove the contrapositive. The negation of property (2) implies that there is an $r_{1}>0$ such that all points in $r_{1}$ open neighborhoods of $a_{\omega}$ and $b_{\omega}$ respectively have infinite or undefined $\mathbb{S}_{\omega}(S)$ distance. Recall that $\mathcal{P}_{\omega}(S)$ is locally path connected. Let $r_{2}>0$ be a constant such that the $r_{2}$ open neighborhoods of $a_{\omega}$ 
and $b_{\omega}$ are path connected. Set $3 r=\min \left(r_{1}, r_{2}\right)$. By choosing $r_{1}$ to be sufficiently small, we can assume that $d_{\mathcal{P}_{\omega}(S)}\left(a_{\omega}, b_{\omega}\right)>6 r$.

Let the sequences $\left(a_{i}^{\prime}\right),\left(b_{i}^{\prime}\right)$ represent any points $a_{\omega}^{\prime}, b_{\omega}^{\prime}$ in $r$ neighborhoods of $a_{\omega}$, $b_{\omega}$ respectively, let $\gamma_{i}$ be a hierarchy path between $a_{i}^{\prime}$ and $b_{i}^{\prime}$, and let $\gamma_{\omega}$ represent its ultralimit. By construction $\gamma_{\omega}$ is a biLipschitz quasi-geodesic. Let $a_{\omega}^{\prime \prime}$ denote the last point on $\gamma_{\omega}$ of distance $r$ from $a_{\omega}$, and let $a_{\omega}^{\prime \prime \prime}$ denote the last point on $\gamma_{\omega}$ of distance $2 r$ from $a_{\omega}$. Similarly, let $b_{\omega}^{\prime \prime}$ denote the last point on $\gamma_{\omega}$ of distance $r$ from $b_{\omega}$, and let $b_{\omega}^{\prime \prime \prime}$ denote the last point on $\gamma_{\omega}$ of distance $2 r$ from $b_{\omega}$. See Figure 2. We will show that the biLipschitz quasi-geodesic $\gamma_{\omega}$ contains a cut-point between the points $a_{\omega}^{\prime \prime}$ and $b_{\omega}^{\prime \prime}$. Then, local path connectedness implies that the cut-point also separates $a_{\omega}$ and $b_{\omega}$, thus completing the proof of the negation of (1).

We will proceed by considering two cases. In the first case we will obtain a cut-point using the machinery of microscopic jets and in the second case we will obtain a cut-point using the machinery of structurally integral corners.

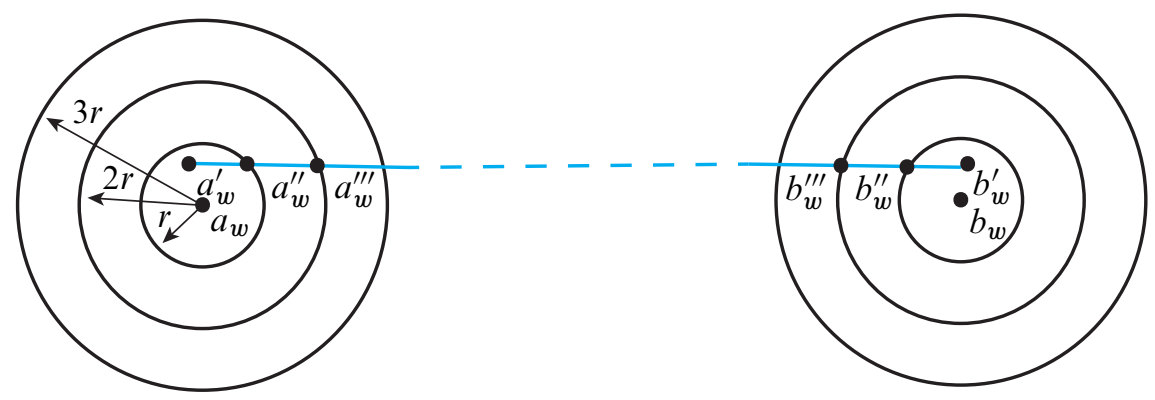

Figure 2: The dotted line is a biLipschitz quasi-geodesic $\gamma_{\omega}$ from $a_{\omega}^{\prime}$ to $b_{\omega}^{\prime}$

Case one Assume there is an $r^{\prime}$ such that for all $a_{\omega}^{0}, b_{\omega}^{0}$ in $3 r^{\prime}$ neighborhoods of $a_{\omega}, b_{\omega}$, with $\left(a_{i}^{0}\right),\left(b_{i}^{0}\right)$ any representatives thereof, respectively, and that there is a $\bar{Y}$ in $\mathcal{N E}^{\omega}(S)$ with

$$
\lim _{\omega} d_{\mathcal{C}\left(Y_{i}\right)}\left(a_{i}^{0}, b_{i}^{0}\right)=\infty
$$

By abuse of notation assume that we have replaced $r$ described above by $r=\min \left\{r, r^{\prime}\right\}$. In particular, since $a_{\omega}^{\prime \prime \prime}, b_{\omega}^{\prime \prime \prime}$ are contained in $3 r$ neighborhoods of $a_{\omega}, b_{\omega}$, respectively, the assumption of the case ensures that for some $\bar{Y} \in \mathcal{N} \mathcal{E}^{\omega}(S)$, we have

$$
\lim _{\omega} d_{\mathcal{C}\left(Y_{i}\right)}\left(a_{i}^{\prime \prime \prime}, b_{i}^{\prime \prime \prime}\right)=\infty
$$


Then, by Theorem 2.14 there is a microscopic jet $J=\left(\bar{g}, \bar{Y}, \overline{a^{\prime \prime \prime}}, \overline{b^{\prime \prime \prime}}\right)$ with $\bar{g} \subset$ $\left.\gamma_{\omega}\right|_{\left[a_{\omega}^{\prime \prime \prime}, b_{\omega}^{\prime \prime \prime}\right]}$ and such that $a_{\omega}^{\prime \prime \prime} \chi_{J} b_{\omega}^{\prime \prime \prime}$. By definition,

$$
\lim _{\omega} d_{\mathcal{C}\left(Y_{i}\right)}\left(\pi_{g_{i}}\left(a_{i}^{\prime \prime}\right), \pi_{g_{i}}\left(b_{i}^{\prime \prime}\right)\right)=\infty
$$

By the properties of hierarchies in Theorem 2.9 it follows that

$$
\lim _{\omega} d_{\mathcal{C}\left(Y_{i}\right)}\left(\pi_{g_{i}}\left(a_{i}^{\prime \prime}\right), \pi_{g_{i}}\left(b_{i}^{\prime \prime}\right)\right)=\infty
$$

and hence $a_{\omega}^{\prime \prime} \chi_{J} b_{\omega}^{\prime \prime}$.

Since the complement $\overline{Y^{c}}$ is the emptyset, $\iota(J) \times \mathcal{P}_{\omega}\left(\overline{Y^{c}}\right)$ is a single point in the asymptotic cone. Moreover, by construction this single point is not equal to either $a_{\omega}^{\prime \prime}$ or $b_{\omega}^{\prime \prime}$. Theorem 2.15 implies that the initial point of the jet is a cut-point between $a_{\omega}^{\prime \prime}$ and $b_{\omega}^{\prime \prime}$. This completes the proof of case one.

Case two Assume the negatation of case one. Then in any neighborhoods of $a_{\omega}, b_{\omega}$ there are $a_{\omega}^{0}, b_{\omega}^{0}$ with representatives $\left(a_{i}^{0}\right),\left(b_{i}^{0}\right)$, such that for all $\bar{Y}$ in $\mathcal{N} \mathcal{E}^{\omega}(S)$ we have

$$
\lim _{\omega} d_{\mathcal{C}\left(Y_{i}\right)}\left(a_{i}^{0}, b_{i}^{0}\right)<\infty .
$$

For $r$ neighborhoods of $a_{\omega}, b_{\omega}$ set the points $a_{\omega}^{0}, b_{\omega}^{0}$ with representatives $\left(a_{i}^{0}\right),\left(b_{i}^{0}\right)$, guaranteed to exist by the hypothesis of the case to be equal to $a_{\omega}^{\prime}, b_{\omega}^{\prime}$, with representatives $\left(a_{i}^{\prime}\right),\left(b_{i}^{\prime}\right)$, respectively. Then as above, let $\gamma_{i}$ be a hierarchy path between $a_{i}^{\prime}$ and $b_{i}^{\prime}$, and similarly define the points $a_{i}^{\prime \prime}, a_{i}^{\prime \prime \prime}, b_{i}^{\prime \prime}, b_{i}^{\prime \prime \prime}$. By the assumptions of the case the hierarchies $\gamma_{i}$ have the property that for all $Y \in \mathcal{N E}(S)$, the projection of $\gamma_{i}$ to $\mathcal{C}(Y)$ is uniformly bounded. In particular, the hierarchies $\gamma_{i}$ have uniformly bounded main geodesic length and travels for uniformly bounded distances in all connected non-separating essential subsurfaces $Y$. By Lemma 2.1 there is a $k$ such that $\omega$-as the main geodesic in $\gamma_{i}$ has length exactly $k$. Specifically, $\omega$-as there is a tight main geodesic in $\mathcal{C}(S)$, with simplices $g_{0 i}, \ldots, g_{k i}$ such that $g_{0 i} \subset a_{i}^{\prime}, g_{k i} \subset b_{i}^{\prime}$. By construction, the hierarchy $\gamma_{i}$ travels through the finite set of natural product regions, $\mathcal{Q}\left(g_{0 i}\right), \ldots, \mathcal{Q}\left(g_{k i}\right)$. See Figure 3 .

Without loss of generality we can assume that for all $j$, either $\gamma_{j i} \in \mathcal{P}(S)$, ie $\gamma_{j i}$ is an entire pants decomposition of a surface, or for any $\left(W_{i}\right)$ a sequence of connected essential subsurfaces in the complement $S \backslash g_{j i}$, we have $\lim _{\omega} d_{\mathcal{C}\left(W_{i}\right)}\left(a_{i}^{\prime}, b_{i}^{\prime}\right)=\infty$. If not, by iterating the argument we used above for a finite length $\mathcal{C}(S)$ main geodesic we can $\omega$-as replace the multicurve $g_{j i}$ by a finite list of connected simplices in $\mathcal{C}(S)$ each containing $g_{j i}$ as a proper multicurve. This iteration process of replacing a multicurve $g_{j i}$ from our finite list $\left\{g_{0 i}, \ldots, g_{k i}\right\}$ with finite sequences of multicurves each containing the original multicurve as a proper multicurve must terminate due to the 


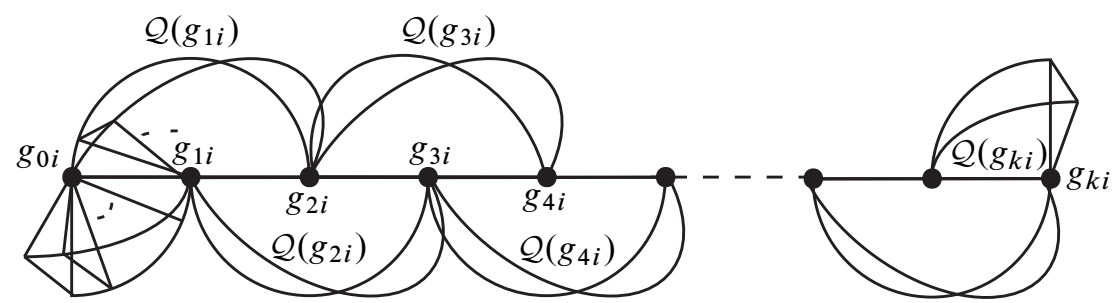

Figure 3: The ultralimit of hierarchy paths with a uniformly bounded main geodesics. Notice that each of the vertices along the finite length main geodesic are separating multicurves.

finite complexity of the surface $S$. Accordingly, we have a finite list of natural product regions and singletons through which our hierarchy path $\gamma_{i}$ from $a_{i}^{\prime}$ to $b_{i}^{\prime} \omega$-as travels. Since the list of natural product regions and singletons is bounded $\omega$-as, coarsely we can ignore the singletons. That is, coarsely our hierarchy path $\gamma_{i}$ from $a_{i}^{\prime}$ to $b_{i}^{\prime}$ $\omega$-as travels through only a finite list of natural product regions, $\mathcal{Q}\left(g_{0 i}\right), \ldots, \mathcal{Q}\left(g_{k^{\prime} i}\right)$ such that for any $\left(W_{i}\right)$ a sequence of connected component of $S \backslash g_{j i}$, we have $\lim _{\omega} d_{\mathcal{C}\left(W_{i}\right)}\left(a_{i}^{\prime}, b_{i}^{\prime}\right)=\infty$. By the assumptions of our case, for each $j, \omega-$ as $g_{i, j}$ is a separating multicurve, or equivalently for each $j$ the region $\mathcal{Q}\left(g_{j i}\right)$ is a natural product region. Moreover, by construction for all $\left.\left.j, \lim _{\omega} d_{\mathcal{P}(S)}\left(g_{i j}\right\lrcorner g_{(i+1) j}, g_{(i+1) j}\right\lrcorner g_{i j},\right)$ is bounded. Notice that all of the above analysis holds after restricting to the subbiLipschitz quasi-geodesic $\left.\gamma_{i}\right|_{a_{i}^{\prime \prime \prime}, b_{i}^{\prime \prime \prime}}$. Assume we have done so.

However, by the negation of condition (2) of the theorem, it follows that there are consecutive separating multicurves, $g_{j i}, g_{(j+1) i}$ in our list such that

$$
\lim _{\omega} d_{\mathbb{S}(S)}\left(g_{j i}, g_{(j+1), i}\right)=\infty .
$$

In particular, in conjunction with the analysis of the previous paragraph, we have a structurally integral corner $\overline{g_{j}} C_{\overline{g_{j+1}}}$. By construction,

$$
a_{\omega}^{\prime \prime}, b_{\omega}^{\prime \prime} \neq \overline{g_{j}^{\prime}} C_{\overline{g_{j+1}^{\prime}}}
$$

as the corner is on the biLipschitz quasi-geodesic $\left.\gamma_{\omega}\right|_{\left[a_{\omega}^{\prime \prime \prime}, b_{\omega}^{\prime \prime \prime}\right]}$. Furthermore,

$$
a_{\omega}^{\prime \prime} \chi_{\overline{g_{j}^{\prime}}}, \overline{g_{j+1}^{\prime}} b_{\omega}^{\prime \prime}
$$

as by our assumptions $a_{\omega}^{\prime \prime}$ is in case one of the equivalence relation $\sim \overline{g_{j}^{\prime}}, \overline{g_{j+1}^{\prime}}$ while $b_{\omega}^{\prime \prime}$ is in case two of the equivalence relation $\sim \overline{g_{j}^{\prime}} \overline{g_{j+1}^{\prime}}$. Corollary 4.7 implies that the structurally integral corner $\overline{g_{j}^{\prime}} C \overline{g_{j+1}^{\prime}}$ is a cut-point between the points $a_{\omega}^{\prime \prime}, b_{\omega}^{\prime \prime}$. This completes the proof of the theorem. 
In view of the characterizations of hyperbolicity and relative hyperbolicity in terms of asymptotic cones [12; 14], Theorem 4.8 generalizes results of Brock and Farb [8] and Brock and Masur [9] proving that Teichmüller spaces of complexity one, two are hyperbolic, relatively hyperbolic as in those cases $\mathcal{S}(S)$ is trivial, totally disconnected respectively.

\section{Thickness of Teichmüller spaces}

In this section we focus our analysis on the surface $S_{2,1}$ which has previously proven to be difficult to understand, as is apparent from the surrounding literature. In particular, we complete the thickness classification of Teichmüller space of all surfaces of finite type presented in Table 1. Specifically, we prove that the Teichmüller space of the surface $S_{2,1}$ is thick of order two, thereby answering questions of [3;9]. Throughout this section we will use the pants complex as a quasi-isometric model for Teichmüller space, often making statements and theorems about Teichmüller space with proofs obtained from considering the pants complex.

\begin{tabular}{c|ccccc}
$\vdots$ & $\vdots$ & $\vdots$ & $\vdots$ & $\vdots$ & $\vdots$ \\
7 & $\mathrm{~T} 1$ & $\mathrm{~T} 1$ & $\mathrm{~T} 1$ & $\mathrm{~T} 1$ & $\ldots$ \\
6 & $\mathrm{RH}$ & $\mathrm{T} 1$ & $\mathrm{~T} 1$ & $\mathrm{~T} 1$ & $\ldots$ \\
5 & $\mathrm{H}$ & $\mathrm{T} 1$ & $\mathrm{~T} 1$ & $\mathrm{~T} 1$ & $\ldots$ \\
4 & $\mathrm{H}$ & $\mathrm{T} 1$ & $\mathrm{~T} 1$ & $\mathrm{~T} 1$ & $\ldots$ \\
3 & & $\mathrm{RH}$ & $\mathrm{T} 1$ & $\mathrm{~T} 1$ & $\ldots$ \\
2 & & $\mathrm{H}$ & $\mathrm{T} 1$ & $\mathrm{~T} 1$ & $\ldots$ \\
1 & & $\mathrm{H}$ & $\mathrm{T} 2$ & $\mathrm{~T} 1$ & $\ldots$ \\
0 & & & $\mathrm{RH}$ & $\mathrm{T} 1$ & $\ldots$ \\
\hline$n \uparrow g \rightarrow$ & 0 & 1 & 2 & 3 & $\ldots$
\end{tabular}

Table 1: Hyperbolicity/thickness classification of Teichmüller spaces for all surfaces. $\mathrm{H}=$ hyperbolic, $\mathrm{RH}=$ relatively hyperbolic, $\mathrm{T} 1=$ thick of order one, and $\mathrm{T} 2=$ thick of order two.

\section{$5.1 \mathcal{T}\left(S_{2,1}\right)$ is thick of order one or two}

Theorem 5.1 [1, Theorem 7.1] Let $\gamma$ be any pseudo-Anosov axis in $\mathcal{P}(S)$, and let $\gamma_{\omega}$ be its ultralimit in any asymptotic cone $\mathcal{P}_{\omega}(S)$. Then any distinct points on $\gamma_{\omega}$ are separated by a cut-point. 
Since all mapping class groups of surfaces with positive complexity contain pseudoAnosov elements, and given any pseudo-Anosov axis, one can choose an asymptotic cone in which its ultralimit is non-trivial, by Theorem 5.1 it follows that $\mathcal{T}(S)$ is never wide, and hence never thick of order zero.

On the other hand, presently we will review the argument in [9] proving that $\mathcal{T}\left(S_{2,1}\right)$ is thick of order at most two. Given $\alpha \in \mathcal{C}_{\text {sep }}\left(S_{2,0}\right)$, let $\tilde{\alpha} \in \mathcal{C}_{\text {sep }}\left(S_{2,1}\right)$ denote any lift of $\alpha$ with respect to the projection $\pi=\pi_{\mathcal{C}\left(S_{2,0}\right)}: \mathcal{C}_{\text {sep }}\left(S_{2,1}\right) \rightarrow \mathcal{C}_{\text {sep }}\left(S_{2,0}\right)$ which forgets about the boundary component. By topological considerations $S \backslash \widetilde{\alpha}=Y_{1} \sqcup Y_{2}=S_{1,1} \sqcup S_{1,2}$. Since $\operatorname{diam}\left(\mathcal{P}\left(Y_{i}\right)\right)=\infty$, we can choose bi-infinite geodesics $\rho_{i} \in \mathcal{P}\left(Y_{i}\right)$, and in fact, by Theorem 2.10, the span of any two such bi-infinite geodesics in the different connected components $Y_{1}, Y_{2}$ comprise a quasi-flat. In particular, it follows that the sets $\mathcal{Q}(\widetilde{\alpha})$ are non-trivial product regions, and in particular are wide. Moreover, using the property of hierarchies in Theorem 2.9, it follows that these subsets $\mathcal{Q}(\widetilde{\alpha})$ satisfy the non-triviality property of every point having a bi-infinite quasi-geodesic through it. Hence, the subsets $\mathcal{Q}(\widetilde{\alpha})$ are thick of order zero.

With the notation as above, set

$$
\mathcal{X}(\alpha)=\left\{Q \in \mathcal{P}\left(S_{2,1}\right) \mid \alpha \in \pi(Q)\right\}=\bigcup_{\widetilde{\alpha}} \mathcal{Q}(\widetilde{\alpha}) .
$$

Using the subsets $\mathcal{X}(\alpha)$ as a network of thickly connected thick of order one pieces, [9] prove that $\mathcal{T}\left(S_{2,1}\right)$ is thick of order at most two.

\section{$5.2 \mathcal{T}\left(S_{2,1}\right)$ is thick of order two}

Taking ultra limits of the sets $\mathcal{X}(\alpha)$ in Equation (5-1), we define

$$
\mathcal{X}_{\omega}(\bar{\alpha})=:\left\{x_{\omega} \in \mathcal{P}_{\omega}(S) \mid x_{\omega} \text { has a representative }\left(x_{i}^{\prime}\right) \text { with } x_{i}^{\prime} \in \mathcal{X}\left(\alpha_{i}\right) \omega \text {-as }\right\}
$$

Lemma 5.2 For $\bar{\alpha} \in \mathcal{C}_{\text {sep }}^{\omega}\left(S_{2,0}\right), \mathcal{X}_{\omega}(\bar{\alpha}) \subset \mathcal{P}_{\omega}\left(S_{2,1}\right)$ is a closed set.

Proof Consider the 1-Lipschitz (hence continuous) projection $\pi_{\mathcal{P}_{\omega}\left(S_{2,0}\right)}: \mathcal{P}_{\omega}\left(S_{2,1}\right) \rightarrow$ $\mathcal{P}_{\omega}\left(S_{2,0}\right)$ which takes a representative sequence $\left(a_{i}\right)$ for $a_{\omega}$ and maps it to a representative sequence of $\left(\pi_{\mathcal{P}\left(S_{2,0}\right)}\left(a_{i}\right)\right)$ where the map $\pi_{\mathcal{P}\left(S_{2,0}\right)}: \mathcal{P}\left(S_{2,1}\right) \rightarrow \mathcal{P}\left(S_{2,0}\right)$ is the natural projection which forgets about the boundary component. By definition $\left(\pi_{\mathcal{P}_{\omega}\left(S_{2,0}\right)}\right)^{-1}\left(\mathcal{Q}_{\omega}(\bar{\alpha})\right)=\mathcal{X}_{\omega}(\bar{\alpha})$. By continuity, the result of the lemma follows from the fact that $\mathcal{Q}_{\omega}(\bar{\alpha}) \subset \mathcal{P}_{\omega}\left(S_{2,0}\right)$ is closed.

Recall Lemma 3.3. In light of the notation developed in this section, as a special case we have the following corollary: 
Corollary 5.3 Assume $\bar{\alpha} \neq \bar{\beta} \in \mathcal{C}_{\text {sep }}^{\omega}\left(S_{2,1}\right)$ and let

$$
\mathcal{X}_{\omega}(\bar{\alpha})=\bigcup \mathcal{Q}_{\omega}(\overline{\widetilde{\alpha}}) \quad \text { and } \quad \mathcal{X}_{\omega}(\bar{\beta})=\bigcup \mathcal{Q}_{\omega}(\overline{\widetilde{\beta}}) .
$$

Then

$$
\left|\mathcal{Q}_{\omega}(\overline{\widetilde{\alpha}}) \cap \mathcal{Q}_{\omega}(\overline{\widetilde{\beta}})\right| \leq 1
$$

and moreover, for $\bar{W}, \bar{V} \in \mathcal{E}^{\omega}(S)$ with $\overline{\partial W}=\overline{\widetilde{\alpha}}, \overline{\partial V}=\overline{\widetilde{\beta}}$ we have

$$
\Phi_{\bar{W}, x_{\omega}}\left(\mathcal{Q}_{\omega}(\overline{\widetilde{\beta}})\right)=\{\mathrm{pt}\}, \quad \Phi_{\bar{V}, y_{\omega}}\left(\mathcal{Q}_{\omega}(\overline{\widetilde{\alpha}})\right)=\{\mathrm{pt}\},
$$

where $\Phi_{\bar{W}}, x_{\omega}$ is the projection defined in Equation (2-2).

The next theorem will be used to prove that the ultralimit of any thick of order zero subset $Z$ in $\mathcal{P}\left(S_{2,1}\right)$ must be contained entirely inside a particular single closed set of the form $\mathcal{X}_{\omega}(\bar{\alpha})$. Recall that by definition, a subspace $Z$ is thick of order zero if (i) it is wide, namely in every asymptotic cone $P_{\omega}\left(S_{2,1}\right)$, the subset corresponding to the ultralimit

$$
Z_{\omega}=:\left\{x_{\omega} \in \mathcal{P}_{\omega}\left(S_{2,1}\right) \mid x_{\omega} \text { has a representative sequence }\left(x_{i}^{\prime}\right) \text { with } x_{i}^{\prime} \in Z \omega \text {-as }\right\}
$$

has the property that any two distinct points in $Z_{\omega}$ are not separated by a cut-point, and moreover (ii) $Z$ satisfies the non-triviality condition of every point being distance at most $c$ from a bi-infinite quasi-geodesic in $Z$.

Theorem 5.4 Let $\left(Z_{i}\right) \subset \mathcal{P}\left(S_{2,1}\right)$ be any sequence of subsets, and let $\mathcal{P}_{\omega}\left(S_{2,1}\right)$ be any asymptotic cone such that the ultralimit $Z_{\omega}$ does not have cut-points. Then $Z_{\omega} \subset \mathcal{X}_{\omega}(\bar{\alpha})$, for some $\bar{\alpha} \in \mathcal{C}_{\text {sep }}^{\omega}\left(S_{2,0}\right)$. Moreover, if in any asymptotic cone $\mathcal{P}_{\omega}\left(S_{2,1}\right)$, the ultralimit $Z_{\omega}$ contains at least two points, then there is a unique such $\bar{\alpha}$ satisfying the following condition: in any neighborhoods of $a_{\omega} \neq b_{\omega} \in Z_{\omega}$ there are points $a_{\omega}^{\prime}, b_{\omega}^{\prime}$ with $d_{\mathbb{S}_{\omega}}\left(S_{2,1}\right)\left(a_{\omega}^{\prime}, b_{\omega}^{\prime}\right)$ bounded, and such that each of the natural product regions $\mathcal{Q}_{\omega}(\bar{C}) \in \mathcal{P}_{\omega}(S)$ in a finite $\mathbb{S}_{\omega}\left(S_{2,1}\right)$ chain from $a_{\omega}^{\prime}$ to $b_{\omega}^{\prime}$ are entirely contained in $\mathcal{X}_{\omega}(\bar{\alpha})$.

Before proving Theorem 5.4 we first prove the following lemma.

Lemma 5.5 Let $\left(Z_{i}\right) \subset \mathcal{P}\left(S_{2,1}\right)$ be any sequence of subsets, and let $\mathcal{P}_{\omega}\left(S_{2,1}\right)$ be any asymptotic cone such that the ultralimit $Z_{\omega}$ is non-trivial and does not have cut-points. Then for all $a_{\omega} \neq b_{\omega}$ in $Z_{\omega}$, it follows that $a_{\omega}, b_{\omega} \subset \mathcal{X}_{\omega}(\bar{\alpha})$, for some $\bar{\alpha} \in \mathcal{C}_{\text {sep }}^{\omega}\left(S_{2,0}\right)$. In fact, $\bar{\alpha}$ can be uniquely identified by the following condition: in any neighborhoods of $a_{\omega} \neq b_{\omega} \in Z_{\omega}$ there are points $a_{\omega}^{\prime}, b_{\omega}^{\prime}$ with $d_{\mathbb{S}_{\omega}\left(S_{2,1}\right)}\left(a_{\omega}^{\prime}, b_{\omega}^{\prime}\right)$ bounded, and such that each of the natural product regions $\mathcal{Q}_{\omega}(\bar{C}) \in \mathcal{P}_{\omega}(S)$ in a finite $\mathbb{S}_{\omega}\left(S_{2,1}\right)$ chain from $a_{\omega}^{\prime}$ to $b_{\omega}^{\prime}$ are entirely contained in $\mathcal{X}_{\omega}(\bar{\alpha})$. 
Proof Since $Z_{\omega}$ does not have any cut points, by Theorem 4.8, in any neighborhoods of $a_{\omega}, b_{\omega}$ there are points $a_{\omega}^{\prime}, b_{\omega}^{\prime}$ with $d_{\mathbb{S}_{\omega}\left(S_{2,1}\right)}\left(a_{\omega}^{\prime}, b_{\omega}^{\prime}\right)$ bounded. That is, there is a finite chain of natural product regions $\mathcal{Q}_{\omega}\left(\widetilde{\widetilde{\alpha}}_{1}\right), \ldots, \mathcal{Q}_{\omega}\left(\overline{\widetilde{\alpha}}_{K}\right)$ such that $a_{\omega}^{\prime} \in \mathcal{Q}_{\omega}\left(\overline{\widetilde{\alpha}_{1}}\right)$, $b_{\omega}^{\prime} \in \mathcal{Q}_{\omega}\left(\overline{\widetilde{\alpha}_{K}}\right)$, and $\left|\mathcal{Q}_{\omega}\left(\overline{\widetilde{\alpha}_{j}}\right) \cap \mathcal{Q}_{\omega}\left(\overline{\widetilde{\alpha}_{j+1}}\right)\right| \geq 2$. As suggested by the notation, for all $j \in\{1, \ldots, K\}, \pi_{\mathcal{C}^{\omega}\left(S_{2,0}\right)}\left(\overline{\widetilde{\alpha}}_{j}\right)=\bar{\alpha}$ for some fixed $\bar{\alpha} \in \mathcal{C}^{\omega}\left(S_{2,0}\right)$ where the projection

$$
\pi_{\mathcal{C}^{\omega}\left(S_{2,0}\right)}: \mathcal{C}^{\omega}\left(S_{2,1}\right) \rightarrow \mathcal{C}^{\omega}\left(S_{2,0}\right)
$$

is the extension to the ultrapower of the natural projection map which forgets about the boundary component. In particular, all the natural product regions $\mathcal{Q}_{\omega}\left(\widetilde{\widetilde{\alpha}_{j}}\right)$ in the chain connecting $a_{\omega}^{\prime}, b_{\omega}^{\prime}$ are contained in the set $\mathcal{X}_{\omega}(\bar{\alpha})$.

Since by Lemma 5.2 the sets $\mathcal{X}_{\omega}(\bar{\alpha})$ are closed, in order to complete the proof of the lemma it suffices to show that for all $a_{\omega}^{\prime}, b_{\omega}^{\prime}$ in small enough neighborhoods of $a_{\omega}, b_{\omega}$, respectively, such that $d_{\mathbb{S}_{\omega}\left(S_{2,1}\right)}\left(a_{\omega}^{\prime}, b_{\omega}^{\prime}\right)$ is bounded, we have that $a_{\omega}^{\prime}$ and $b_{\omega}^{\prime}$ are all always contained in the same set $\mathcal{X}_{\omega}(\bar{\alpha})$ as above. Assume not, that is, assume that in any neighborhoods of $a_{\omega}, b_{\omega}$ there are points $a_{\omega}^{1}, b_{\omega}^{1}$ and $a_{\omega}^{2}, b_{\omega}^{2}$ such that $d_{\mathbb{S}_{\omega}\left(S_{2,1}\right)}\left(a_{\omega}^{1}, b_{\omega}^{1}\right)<\infty$ and $d_{\mathbb{S}_{\omega}\left(S_{2,1}\right)}\left(a_{\omega}^{2}, b_{\omega}^{2}\right)<\infty$, yet $a_{\omega}^{1}, b_{\omega}^{1} \in \mathcal{X}_{\omega}(\bar{\alpha})$ while $a_{\omega}^{2}, b_{\omega}^{2} \in \mathcal{X}_{\omega}(\bar{\beta})$ where $\bar{\alpha} \neq \bar{\beta}$. In particular, we can assume that $a_{\omega}^{1}, b_{\omega}^{1}$ lie in an $r$-neighborhood of $a_{\omega}$ and $a_{\omega}^{2}, b_{\omega}^{2}$ lie in an $r$-neighborhood of $b_{\omega}$ where $r \geq 0$ is a constant such that open $r$-neighborhoods of $a_{\omega}, b_{\omega}$ are path connected. In addition, we can assume that $2 r<d_{\mathcal{P}_{\omega}(S)}\left(a_{\omega}, b_{\omega}\right)$. See Figure 4 for an illustration of this.

Let $\mathcal{Q}_{\omega}\left(\overline{\widetilde{\alpha}_{1}}\right), \ldots, \mathcal{Q}_{\omega}\left(\overline{\widetilde{\alpha}_{m}}\right)$ be a finite chain of product regions in $\mathcal{X}_{\omega}(\bar{\alpha})$ connecting $a_{\omega}^{1}$ and $b_{\omega}^{1}$. Moreover, as in Theorem 4.8 there is a biLipschitz quasi-geodesic path $\rho_{\omega}^{1}$, the ultralimit of hierarchy paths, through the product regions connecting $a_{\omega}^{1}$ and $b_{\omega}^{1}$. Similarly, let $\mathcal{Q}_{\omega}\left({\widetilde{\beta_{1}}}_{1}\right), \ldots, \mathcal{Q}_{\omega}\left({\widetilde{\beta_{n}}}_{n}\right)$ be a finite chain of natural product regions in $\mathcal{X}_{\omega}(\bar{\beta})$ connecting $a_{\omega}^{2}$ and $b_{\omega}^{2}$, and let $\rho_{\omega}^{2}$ be a biLipschitz quasi-geodesic path through the product regions connecting $a_{\omega}^{2}$ and $b_{\omega}^{2}$. By omitting product regions as necessary and using properties of hierarchies in Theorem 2.9 we can assume that initial product region $\mathcal{Q}_{\omega}\left(\overline{\widetilde{\alpha}_{1}}\right)$ of the path $\rho_{\omega}^{1}$ has the property that $\rho_{\omega}^{1}$ exits the product region $\mathcal{Q}_{\omega}\left(\overline{\widetilde{\alpha}_{1}}\right)$ once at a point $e_{\omega} \neq a_{\omega}^{1}$. By Lemma 2.13, there is some $\bar{W} \in \mathcal{S E}^{\omega}(S)$ which is $\omega$-as a connected component of $\overline{S \backslash \widetilde{\alpha}_{1}}$, such that $\pi_{\mathcal{P}_{\omega}(\bar{W})}\left(a_{\omega}^{1}\right) \neq \pi_{\mathcal{P}_{\omega}(\bar{W})}\left(e_{\omega}\right) \in F_{\bar{W}, a_{\omega}^{1}}$.

By our assumptions, $a_{\omega}^{1}$ and $a_{\omega}^{2}$ are connected by a path that remains entirely inside an $r$-neighborhood of $a_{\omega}$. Let $\left[a_{\omega}^{1}, a_{\omega}^{2}\right]$ denote such a path. Similarly, let $\left[b_{\omega}^{1}, b_{\omega}^{2}\right]$ denote a path between the points $b_{\omega}^{1}$ and $b_{\omega}^{2}$. We can assume that $\left(a_{\omega}^{1}, a_{\omega}^{2}\right]$ and $\left(b_{\omega}^{1}, b_{\omega}^{2}\right]$ are contained in $\mathcal{P}_{\omega}\left(S_{2,1}\right) \backslash \mathcal{Q}_{\omega}\left(\overline{\widetilde{\alpha}_{1}}\right)$. If not, we can replace $a_{\omega}^{1}$ and/or $b_{\omega}^{1}$ with points closer to $a_{\omega}^{2}$ and/or $b_{\omega}^{2}$ respectively such that this is the case.

Consider the closed pentagon $P$ with vertices $\left\{a_{\omega}^{1}, e_{\omega}, b_{\omega}^{1}, b_{\omega}^{2}, a_{\omega}^{2}\right\}$ and edges

$$
\left.\rho_{\omega}^{1}\right|_{\left[a_{\omega}^{1}, e_{\omega}\right]},\left.\quad \rho_{\omega}^{1}\right|_{\left[e_{\omega}, b_{\omega}^{1}\right]}, \quad\left[b_{\omega}^{1}, b_{\omega}^{2}\right], \quad \rho_{\omega}^{2}, \quad\left[a_{\omega}^{1}, a_{\omega}^{2}\right] .
$$




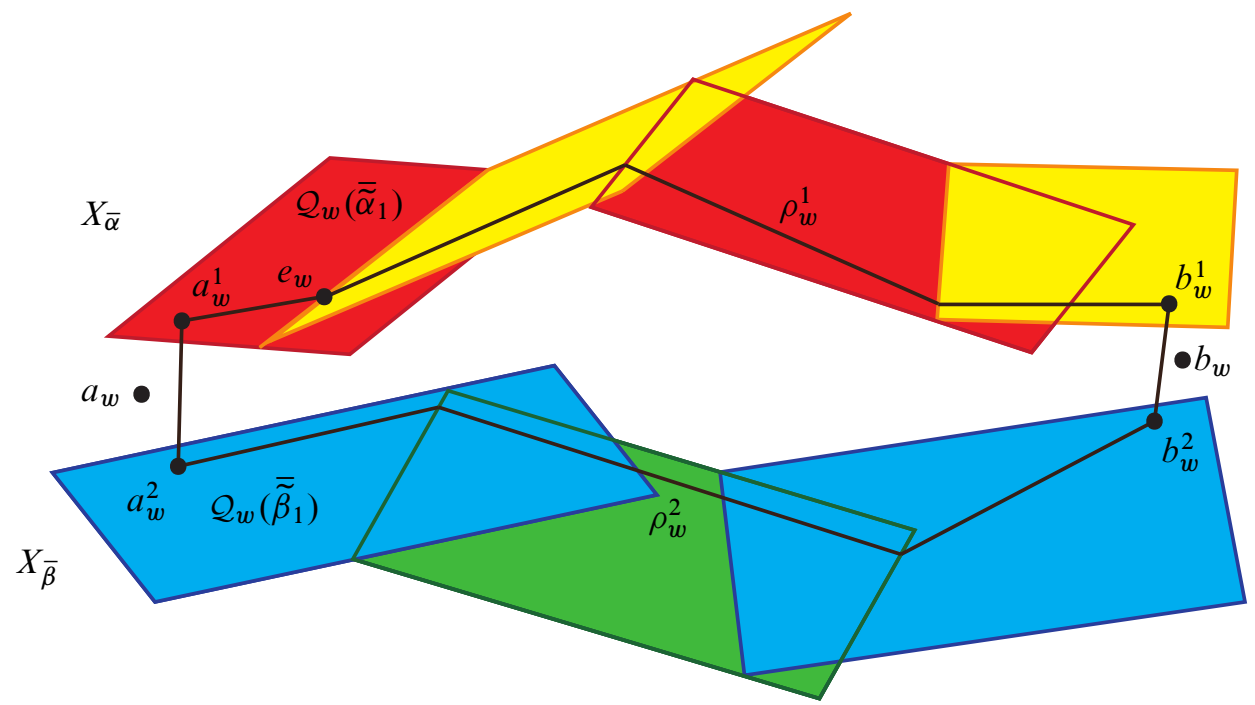

Figure 4: In neighborhoods of $a_{\omega}, b_{\omega}$ there are points $a_{\omega}^{1}, b_{\omega}^{1}$ and $a_{\omega}^{2}, b_{\omega}^{2}$, respectively, such that $d_{\mathbb{S}_{\omega}(S)}\left(a_{\omega}^{1}, b_{\omega}^{1}\right)<\infty, d_{\mathbb{S}_{\omega}(S)}\left(a_{\omega}^{2}, b_{\omega}^{2}\right)<\infty$, yet $a_{\omega}^{1}, b_{\omega}^{1} \in \mathcal{X}_{\omega}(\bar{\alpha})$ while $a_{\omega}^{2}, b_{\omega}^{2} \in \mathcal{X}_{\omega}(\bar{\beta})$ where $\bar{\alpha} \neq \bar{\beta}$. This situation cannot occur in $\mathcal{P}_{\omega}\left(S_{2,1}\right)$.

It should be noted that some sides of the pentagon may be trivial, although this does not affect the argument. Applying the continuous projection $\Phi_{\bar{W}}, x_{\omega}$ of Theorem 2.12 to the pentagon $P$, we have $\Phi_{\bar{W}, x_{\omega}}\left(e_{\omega}\right)=\Phi_{\bar{W}, x_{\omega}}\left(b_{\omega}^{1}\right)=\Phi_{\bar{W}, x_{\omega}}\left(b_{\omega}^{2}\right)$. Similarly, $\Phi \bar{W}, x_{\omega}\left(a_{\omega}^{1}\right)=\Phi_{\bar{W}, x_{\omega}}\left(a_{\omega}^{2}\right)$ as by construction the edges $\left.\rho_{\omega}^{1}\right|_{\left[e_{\omega}, b_{\omega}^{1}\right]},\left[b_{\omega}^{1}, b_{\omega}^{2}\right]$ and $\left[a_{\omega}^{1}, a_{\omega}^{2}\right]$ are contained in $\mathcal{P}_{\omega}\left(S_{2,1}\right) \backslash P_{\bar{W}, x_{\omega}}$. Furthermore, by Corollary 5.3 and continuity of the projection, $\Phi_{\bar{W}}, x_{\omega}\left(\rho_{\omega}^{2}\right)$ is a single point and is in fact equal to $\Phi_{\bar{W}, x_{\omega}}\left(a_{\omega}^{2}\right)=\Phi_{\bar{W}, x_{\omega}}\left(b_{\omega}^{2}\right)$. Putting things together we have

$$
\Phi_{\bar{W}, x_{\omega}}\left(e_{\omega}\right)=\Phi_{\bar{W}, x_{\omega}}\left(b_{\omega}^{1}\right)=\Phi_{\bar{W}, x_{\omega}}\left(b_{\omega}^{2}\right)=\Phi_{\bar{W}, x_{\omega}}\left(a_{\omega}^{2}\right)=\Phi_{\bar{W}, x_{\omega}}\left(a_{\omega}^{1}\right) .
$$

However, this is a contradiction to our assumption that $\Phi_{\bar{W}, x_{\omega}}\left(a_{\omega}^{1}\right) \neq \Phi_{\bar{W}, x_{\omega}}\left(e_{\omega}\right)$, thus completing the proof.

Using the proof of Lemma 5.5, presently we prove Theorem 5.4.

Proof of Theorem 5.4 By Lemma 5.5 we know that given any two distinct points $a_{\omega}, b_{\omega} \in Z_{\omega}$, the points $a_{\omega}, b_{\omega}$ are contained in a common subset $\mathcal{X}_{\omega}(\bar{\alpha})$ where $\bar{\alpha} \in \mathcal{C}_{\text {sep }}^{\omega}\left(S_{2,0}\right)$ is such that in any neighborhoods of $a_{\omega} \neq b_{\omega} \in Z_{\omega}$ there are points $a_{\omega}^{\prime}, b_{\omega}^{\prime}$ with $d_{\mathbb{S}_{\omega}\left(S_{2,1}\right)}\left(a_{\omega}^{\prime}, b_{\omega}^{\prime}\right)$ bounded, and such that each of the natural product 
regions $\mathcal{Q}_{\omega}(\bar{C})$ in a finite $\mathbb{S}_{\omega}\left(S_{2,1}\right)$ chain from $a_{\omega}^{\prime}$ to $b_{\omega}^{\prime}$ are entirely contained in $\mathcal{X}_{\omega}(\bar{\alpha})$.

Let $c_{\omega} \in Z_{\omega}$ be any third point in $Z_{\omega}$, (possibly the same as $a_{\omega}$ or $b_{\omega}$ ). Similarly, it follows that the points $a_{\omega}, c_{\omega}\left(b_{\omega}, c_{\omega}\right)$ are contained in a common subset $\mathcal{X}_{\omega}(\bar{\beta})$ $\left(\mathcal{X}_{\omega}(\bar{\gamma})\right)$ where $\bar{\beta}(\bar{\gamma})$ is an element of $\mathcal{C}_{\text {sep }}^{\omega}\left(S_{2,0}\right)$ such that in any neighborhoods of $a_{\omega}$ and $c_{\omega}\left(b_{\omega}\right.$ and $\left.c_{\omega}\right)$ there are points $a_{\omega}^{\prime}, c_{\omega}^{\prime}\left(b_{\omega}^{\prime}, c_{\omega}^{\prime}\right)$ with $d_{\mathbb{S}_{\omega}\left(S_{2,1}\right)}\left(a_{\omega}^{\prime}, c_{\omega}^{\prime}\right)$ bounded $\left(d_{\mathbb{S}_{\omega}}\left(S_{2,1}\right)\left(b_{\omega}^{\prime}, c_{\omega}^{\prime}\right)\right.$ bounded), and such that each of the natural product regions $\mathcal{Q}_{\omega}(\bar{C}) \in \mathcal{P}_{\omega}(S)$ in a finite $\mathbb{S}_{\omega}\left(S_{2,1}\right)$ chain from $a_{\omega}^{\prime}$ to $c_{\omega}^{\prime}$ ( $b_{\omega}^{\prime}$ to $c_{\omega}^{\prime}$ ) are entirely contained in $\bar{\beta}(\bar{\gamma})$. But then, considering the triangle between the points $a_{\omega}^{\prime}, b_{\omega}^{\prime}, c_{\omega}^{\prime}$ and using the same projection arguments in Lemma 5.5 to generalize the contradiction argument with the pentagon, it follows that $\bar{\alpha}=\bar{\beta}=\bar{\gamma}$. Notice if $c_{\omega}$ is the same as $a_{\omega}$ or $b_{\omega}$, the proof is identical to the proof in Lemma 5.5.

Since $c_{\omega}$ is arbitrary, it follows that $Z_{\omega} \subset \mathcal{X}_{\omega}(\bar{\alpha})$ where $\bar{\alpha}$ is uniquely determined by the property described in the statement of the theorem.

As a corollary of the proof of Lemma 5.5, we have the following:

Corollary 5.6 Let $\left(Z_{i}\right),\left(Z_{i}^{\prime}\right) \subset \mathcal{P}\left(S_{2,1}\right)$ be any sequences of subsets and $\mathcal{P}_{\omega}\left(S_{2,1}\right)$ be an asymptotic cone such that $Z_{\omega}, Z_{\omega}^{\prime} \subset \mathcal{P}_{\omega}\left(S_{2,1}\right)$ each one contains at least two points, and each one has no cut-points. As in Theorem 5.4 assume that $Z_{\omega} \subset \mathcal{X}_{\omega}(\bar{\alpha})$ and $Z_{\omega}^{\prime} \subset \mathcal{X}_{\omega}(\bar{\beta})$ for some $\bar{\alpha}, \bar{\beta} \in \mathcal{C}_{\mathrm{sep}}^{\omega}\left(S_{2,0}\right)$, such that $\omega$-as $\alpha_{i} \neq \beta_{i}$, then

$$
\left|Z_{\omega} \cap Z_{\omega}^{\prime}\right| \leq 1 \text {. }
$$

In particular, in the case where the subsets $\left(Z_{i}\right)=\bar{Z}$ and $\left(Z_{i}^{\prime}\right)=\overline{Z^{\prime}}$ are constant, then $Z$ and $Z^{\prime}$ have bounded coarse intersection.

Proof We will show $\left|Z_{\omega} \cap Z_{\omega}^{\prime}\right| \leq 1$ by contradiction. That is, assume $a_{\omega} \neq b_{\omega} \in\left(Z_{\omega} \cap\right.$ $\left.Z_{\omega}^{\prime}\right)$. By Theorem 4.8, in any neighborhoods of $a_{\omega}, b_{\omega}$ there are points $a_{\omega}^{1}, b_{\omega}^{1}$ and $a_{\omega}^{2}, b_{\omega}^{2}$, such that $d_{\mathbb{S}_{\omega}\left(S_{2,1}\right)}\left(a_{\omega}^{1}, b_{\omega}^{1}\right)<\infty$ and $d_{\mathbb{S}_{\omega}\left(S_{2,1}\right)}\left(a_{\omega}^{2}, b_{\omega}^{2}\right)<\infty$, yet $a_{\omega}^{1}, b_{\omega}^{1} \in$ $\mathcal{X}_{\omega}(\bar{\alpha})$ while $a_{\omega}^{2}, b_{\omega}^{2} \in \mathcal{X}_{\omega}(\bar{\beta})$ where $\bar{\alpha} \neq \bar{\beta}$. Precisely this situation was shown to be impossible in the proof of Lemma 5.5.

Finally, the "in particular" clause of the corollary follows from Lemma 2.2.

Theorem $5.7 \mathcal{T}\left(S_{2,1}\right)$ is thick of order two.

Proof Since thickness is a quasi-isometry invariant property [3] it suffices to prove that $\mathcal{P}\left(S_{2,1}\right)$ is thick of order two. In Section 5.1 we showed that $\mathcal{P}\left(S_{2,1}\right)$ is thick of order at most two and at least one. Hence, it suffices to show that $\mathcal{P}\left(S_{2,1}\right)$ is not 
thick of order one. In fact, we will show that any thick of order one subset is entirely contained inside a non-trivially proper subset of the entire pants complex (that is, a subset which has infinite Hausdorff distance from the entire pants complex).

Fix an asymptotic cone $\mathcal{P}_{\omega}\left(S_{2,1}\right)$ with a constant base point and scaling sequence $s_{i}$. Note that since $\mathcal{P}\left(S_{2,1}\right)$ is connected, for any $q \in \mathcal{P}\left(S_{2,1}\right)$, the constant sequence $\bar{q}$ all represent the same base point of the asymptotic cone $\mathcal{P}_{\omega}\left(S_{2,1}\right)$.

Let $Z$ be any thick of order zero subset in $\mathcal{P}\left(S_{2,1}\right)$. By hypothesis, $Z$ coarsely contains a bi-infinite quasi-geodesic through any point. Fix some point $z \in Z$, and some quasi-geodesic ray $\gamma$ beginning near $z$ and remaining in $Z$. Then for every $s_{i}$, set $y_{i}=\gamma\left(s_{i}\right) \in Z$. By construction, in the asymptotic cone the sequences $\bar{z}$ and $\left(y_{i}\right)$ represent distinct points contained in $Z_{\omega} \subset \mathcal{P}_{\omega}\left(S_{2,1}\right)$. In particular, we have just shown that every thick of order zero subset $Z \subset \mathcal{P}\left(S_{2,1}\right)$ has ultralimit $Z_{\omega}$ containing at least two distinct points in the asymptotic cone $\mathcal{P}\left(S_{2,1}\right)$. By Theorem 5.4 it follows that every thick of order zero subset $Z$ in $\mathcal{P}\left(S_{2,1}\right)$ can be assigned a unique element $\bar{\alpha} \in \mathcal{C}_{\text {sep }}^{\omega}\left(S_{2,0}\right)$. Moreover, Corollary 5.6 implies that a necessary condition for any two thick of order zero subsets $Z, Z^{\prime}$ to be thickly chained together, as in Definition 2.4(ii), is that the two thick of order zero subsets $Z, Z^{\prime}$ are assigned the same element $\bar{\alpha} \in \mathcal{C}_{\text {sep }}^{\omega}\left(S_{2,1}\right)$.

It follows that any thick of order one subset $Y$ of the space $\mathcal{P}\left(S_{2,1}\right)$ can consist of at most the union of thick of order zero subsets with the same labels $\bar{\alpha} \in \mathcal{C}_{\text {sep }}^{\omega}\left(S_{2,0}\right)$. Hence, the ultralimit $Y_{\omega}$ in the asymptotic cone $\mathcal{P}_{\omega}\left(S_{2,1}\right)$ is entirely contained inside the subset $\mathcal{X}_{\omega}(\bar{\alpha})$ which we will see is a proper subset of $\mathcal{P}_{\omega}\left(S_{2,1}\right)$. The proof of the theorem then follows from the observation that if a subset $Y \subset X$ has finite Hausdorff distance from $X$, then in any asymptotic cone the ultralimit $Y_{\omega}=X_{\omega}$.

To see that $\mathcal{X}_{\omega}(\bar{\alpha})$ is a proper subset of $\mathcal{P}_{\omega}\left(S_{2,1}\right)$, notice that under the surjective projection $\pi: \mathcal{P}_{\omega}\left(S_{2,1}\right) \rightarrow \mathcal{P}_{\omega}\left(S_{2,0}\right)$, the subset $\mathcal{X}_{\omega}(\bar{\alpha})$ is mapped into the natural product region $\mathcal{Q}_{\omega}(\bar{\alpha})$, a proper subset of $\mathcal{P}_{\omega}\left(S_{2,0}\right)$.

\section{References}

[1] J A Behrstock, Asymptotic geometry of the mapping class group and Teichmüller space, Geom. Topol. 10 (2006) 1523-1578 MR2255505

[2] J Behrstock, R Charney, Divergence and quasimorphisms of right-angled Artin groups, Math. Ann. 352 (2012) 339-356 MR2874959

[3] J Behrstock, C Druțu, L Mosher, Thick metric spaces, relative hyperbolicity, and quasi-isometric rigidity, Math. Ann. 344 (2009) 543-595 MR2501302 
[4] J Behrstock, C Druțu, M Sapir, Median structures on asymptotic cones and homomorphisms into mapping class groups, Proc. Lond. Math. Soc. 102 (2011) 503-554 MR2783135

[5] J Behrstock, B Kleiner, Y Minsky, L Mosher, Geometry and rigidity of mapping class groups, Geom. Topol. 16 (2012) 781-888 MR2928983

[6] J A Behrstock, Y N Minsky, Dimension and rank for mapping class groups, Ann. of Math. 167 (2008) 1055-1077 MR2415393

[7] J F Brock, The Weil-Petersson metric and volumes of 3-dimensional hyperbolic convex cores, J. Amer. Math. Soc. 16 (2003) 495-535 MR1969203

[8] J Brock, B Farb, Curvature and rank of Teichmüller space, Amer. J. Math. 128 (2006) 1-22 MR2197066

[9] J Brock, H Masur, Coarse and synthetic Weil-Petersson geometry: Quasi-flats, geodesics and relative hyperbolicity, Geom. Topol. 12 (2008) 2453-2495 MR2443970

[10] J Brock, H Masur, Y Minsky, Asymptotics of Weil-Petersson geodesic, I: Ending laminations, recurrence, and flows, Geom. Funct. Anal. 19 (2010) 1229-1257 MR2585573

[11] J Brock, H Masur, Y Minsky, Asymptotics of Weil-Petersson geodesics, II: Bounded geometry and unbounded entropy, Geom. Funct. Anal. 21 (2011) 820-850 MR2827011

[12] C Druțu, Quasi-isometry invariants and asymptotic cones, Internat. J. Algebra Comput. 12 (2002) 99-135 MR1902363

[13] C Druţu, M Kapovich, Lectures on the geometric group theory (2013) to be published in the series Amer. Math. Soc. Colloq. Publ. Available at https:// www.math.ucdavis.edu/ kapovich/EPR/kapovich_drutu.pdf

[14] C Druțu, M Sapir, Tree-graded spaces and asymptotic cones of groups, Topology 44 (2005) 959-1058 MR2153979

[15] A Hatcher, W Thurston, A presentation for the mapping class group of a closed orientable surface, Topology 19 (1980) 221-237 MR579573

[16] I Kra, On the Nielsen-Thurston-Bers type of some self-maps of Riemann surfaces, Acta Math. 146 (1981) 231-270 MR611385

[17] J Mangahas, Uniform uniform exponential growth of subgroups of the mapping class group, Geom. Funct. Anal. 19 (2010) 1468-1480 MR2585580

[18] H A Masur, Y N Minsky, Geometry of the complex of curves, I: Hyperbolicity, Invent. Math. 138 (1999) 103-149 MR1714338

[19] H A Masur, Y N Minsky, Geometry of the complex of curves, II: Hierarchical structure, Geom. Funct. Anal. 10 (2000) 902-974 MR1791145

[20] S Schleimer, Notes on the complex of curves (2005) Available at http:// www. warwick.ac.uk/ masgar/Maths/notes.pdf 
[21] H M Sultan, The asymptotic cone of Teichmüller space: Thickness and divergence, $\mathrm{PhD}$ thesis, Columbia University (2012) MR3029689 Available at http:// search . proquest . com/docview/1010998380

[22] H Sultan, Hyperbolic quasi-geodesics in CAT(0) spaces, Geom. Dedicata 169 (2014) 209-224 MR3175245

[23] S A Wolpert, Geodesic length functions and the Nielsen problem, J. Differential Geom. 25 (1987) 275-296 MR880186

Department of Mathematics, Brandeis University

Waltham, MA 02453, USA

HaroldSultan@gmail.com

Received: 24 November 2014 Revised: 22 January 2015 